\title{
Consistent Reconstruction of Cortical Surfaces from Longitudinal Brain MR Images
}

\author{
Gang Li, Jingxin Nie, Guorong Wu, Yaping Wang, Dinggang Shen, and the Alzheimer's \\ Disease Neuroimaging Initiative ${ }^{1}$ \\ Department of Radiology and BRIC, University of North Carolina at Chapel Hill, NC, USA
}

Dinggang Shen: dinggang_shen@med.unc.edu

\begin{abstract}
Accurate and consistent reconstruction of cortical surfaces from longitudinal human brain MR images is of great importance in studying longitudinal subtle change of the cerebral cortex. This paper presents a novel deformable surface method for consistent and accurate reconstruction of inner, central and outer cortical surfaces from longitudinal brain MR images. Specifically, the cortical surfaces of the group-mean image of all aligned longitudinal images of the same subject are first reconstructed by a deformable surface method, which is driven by a force derived from the Laplace's equation. And then the longitudinal cortical surfaces are consistently reconstructed by jointly deforming the cortical surfaces of the group-mean image to all longitudinal images. The proposed method has been successfully applied to two sets of longitudinal human brain MR images. Both qualitative and quantitative experimental results demonstrate the accuracy and consistency of the proposed method. Furthermore, the reconstructed longitudinal cortical surfaces are used to measure the longitudinal changes of cortical thickness in both normal and diseased groups, where the overall decline trend of cortical thickness has been clearly observed. Meanwhile, the longitudinal cortical thickness also shows its potential in distinguishing different clinical groups.
\end{abstract}

\section{Keywords}

Cortical surface reconstruction; longitudinal cortical surface; longitudinal cortical thickness

\section{Introduction}

The human cerebral cortex is a thin, highly folded sheet of gray matter with the thickness varying between 1 and 5 mm (von Economo, 1929; Zilles, 1990; Fischl and Dale, 2000; MacDonald et al., 2000; Han et al., 2004). Reconstruction of cortical surfaces from brain magnetic resonance (MR) images plays a vital role in studying structure and function of human brains (Van Essen et al., 1998; Dale et al., 1999). Having reconstructed cortical

\footnotetext{
(c) 2011 Elsevier Inc. All rights reserved.

Correspondence to: Dinggang Shen, dinggang_shen@med.unc.edu.

${ }^{1}$ Data used in preparation of this article were obtained from the Alzheimer's Disease Neuroimaging Initiative (ADNI) database (www.loni.ucla.edu/ADNI). As such, the investigators within the ADNI contributed to the design and implementation of ADNI and/or provided data but did not participate in analysis or writing of this report. ADNI investigators include (complete listing available at http://adni.loni.ucla.edu/wp1content/uploads/how_to_apply/ADNI_Authorship_List.pdf).

Publisher's Disclaimer: This is a PDF file of an unedited manuscript that has been accepted for publication. As a service to our customers we are providing this early version of the manuscript. The manuscript will undergo copyediting, typesetting, and review of the resulting proof before it is published in its final citable form. Please note that during the production process errors may be discovered which could affect the content, and all legal disclaimers that apply to the journal pertain.
} 
surfaces, one can study the cortical folding patterns of gyri and sulci (Tao et al., 2002; Li et al., 2009; Li et al., 2010a; Li et al., 2010b; Mangin et al., 2010), cortical thickness (Fischl and Dale, 2000; MacDonald et al., 2000; Han et al., 2004), sulcal depth (Van Essen et al., 2001), curvature (Cachia et al., 2003), average convexity (Fischl et al., 1999a), local gyrification (Schaer et al., 2008), surface area, and fiber density (Zhang et al., 2010). Also, one can perform cortical surfaces based spatial normalization (Thompson and Toga, 1996; Fischl et al., 1999b; Van Essen, 2004; Liu et al., 2004), surface-based probabilistic atlas generation (Van Essen and Dierker, 2007), cerebral cortex folding modeling (Nie et al., 2010), functional brain mapping (Van Essen et al., 1998; Andrade et al., 2001) and brain connectivity study (Hagmann et al., 2008), etc. Many methods have been proposed for cortical surface reconstruction from brain MR images in the literature (Mangin et al., 1995; Davatzikos and Bryan, 1996; Xu et al., 1999; Dale et al., 1999; Zeng et al., 1999; Joshi et al., 1999; MacDonald et al., 2000; Van Essen et al., 2001; Kriegeskorte and Goebel, 2001; Shattuck and Leahy, 2002; Han et al., 2004; Kim et al., 2005; Xu et al., 2006; Eskildsen and Ostergaard, 2006; Liu et al., 2008; Shi et al., 2011). However, most existing cortical surface reconstruction methods were mainly designed for working on a single MR image. Recently, longitudinal imaging studies have increasingly received considerable attentions in neuroscience and clinical studies (Reuter and Fischl, 2011). For studying longitudinal changes of cortical structures, which is important to normal development, aging, and disease progression of human brains, it requires more accurate cortical surface reconstruction and representation, since longitudinal cortical changes in short times are usually very subtle, especially in normal aging or even in Alzheimer's disease (Li and Shen, 2011). Therefore, applying the existing cross-sectional methods independently to the reconstruction of cortical surfaces at each time point in a longitudinal imaging study may generate longitudinallyinconsistent cortical surfaces, due to the inconsistency of skull stripping, tissue segmentation, topology correction, surface tessellation and surface evolution, etc. Accordingly, several efforts have been made towards the reconstruction of cortical surfaces from longitudinal human brain MR images (Han et al., 2006; Reuter and Fischl, 2011; Nakamura et al., 2011), e.g., the recent longitudinal processing pipeline in FreeSurfer (Reuter and Fischl, 2011), in which cortical surfaces of the group-mean image (averaged from the rigidly-aligned longitudinal images of a subject) or the median image are used as initialization for each longitudinal image and then separately deformed at each time point to achieve longitudinal cortical surface reconstruction.

The central theme of this paper is to present a new method for accurate and consistent reconstruction of inner, central, and outer cortical surfaces from longitudinal human brain MR images. The inner cortical surface is the interface between white matter (WM) and gray matter (GM), and the outer cortical surface is the interface between GM and cerebrospinal fluid (CSF) (Fischl and Dale, 2000; MacDonald et al., 2000; Han et al., 2004). The central cortical surface is defined as the layer lying in the geometric center of the cortex, approximately corresponding to the cytoarchitechtonic layer four (Xu et al., 1999; Van Essen et al., 2001; Han et al., 2004; Liu et al., 2008). Comparing to the inner and outer cortical surfaces, the central cortical surface could provide better geometric information of the cortex (Xu et al., 1999; Van Essen et al., 2001). For consistent reconstruction of cortical surfaces from longitudinal images, in our method, the cortical surfaces of a group-mean image of all non-rigidly aligned longitudinal images are first reconstructed using a parametric deformable surface method (Kass et al., 1987), which has been extensively adopted for cortical surface reconstruction from a single MR image (Davatzikos and Bryan, 1996; Xu et a., 1999; Dale et al., 1999; Kim et al., 2005; Eskildsen and Ostergaard, 2006; Liu et al., 2008), and then the cortical surfaces of the group-mean image are used as the initialization to reconstruct all longitudinal cortical surfaces simultaneously. To drive the deformable surfaces towards the target surfaces (inner, central and outer cortical surfaces), a force derived from the Laplace's equation (Jones et al., 2000) is adopted. Our proposed 
method has been successfully applied to two sets of longitudinal human brain MR images, and experimental results demonstrate the accuracy and consistency of our proposed method. The reconstructed longitudinal cortical surfaces are used to measure the longitudinal changes of cortical thickness in both normal and diseased groups, and the overall decline trend of cortical thickness in aging has been observed, which is consistent to the findings reported in the literature (Resnick et al., 2000, 2003; Salat et al., 2004; Rettmann et al., 2006; Fjell et al., 2009a, 2009b; Thambisetty et al., 2010). Meanwhile, the longitudinal cortical thickness also shows its potential in distinguishing different clinical groups.

Our proposed method has several advantages over the existing methods (Reuter and Fischl, 2011; Nakamura et al., 2011). First, temporal constraint is incorporated in longitudinal cortical surface reconstruction by jointly deforming cortical surfaces of all longitudinal images simultaneously, in contrast to the existing methods which independently deform the cortical surface from the mean or median image at each time point (Reuter and Fischl, 2011; Nakamura et al., 2011). Second, a force derived from the Laplace's equation (Jones et al., 2000) is used to drive the deformable surface to help preserve the topology of the deformable surface. Third, the group-mean image in our method is obtained by a nonlinear groupwise registration method (Wu et al., 2011), in contrast to the rigid alignment used in the existing methods (Reuter and Fischl, 2011; Nakamura et al., 2011). At last, a longitudinally-consistent tissue segmentation method (Xue et al., 2006) is also adopted to facilitate the longitudinally-consistent cortical surface reconstruction.

\section{Methods}

Given longitudinal brain MR images of a subject, our method for consistent cortical surface reconstruction consists of the following 4 major steps, as shown in Fig. 1. First, longitudinal images are preprocessed, including the following steps: intensity-inhomogeneity correction, rigid alignment, and non-brain-tissues removing. Second, longitudinal images are groupwisely registered to obtain a group-mean image without bias, and also their tissues are consistently segmented into WM, GM, and CSF. Third, the cortical surfaces of the groupmean image are reconstructed using a deformable surface method. Finally, the cortical surfaces of the group-mean image are warped to each longitudinal image and jointly deformed to reconstruct longitudinal cortical surfaces at different time points.

\subsection{Preprocessing}

The preprocessing procedure includes the following steps: (1) correction of intensity inhomogeneity of longitudinal images using N3 (Sled et al., 1998), (2) rigid registration of follow-up images to the corresponding baseline image (the first scan), and rigid registration of the baseline image to an atlas space, and combining the transform matrices in these two steps to a single transform matrix to warp the original follow-up images onto the atlas space accordingly, (3) skull stripping of the baseline images using BET (Smith, 2002) in Oxford FSL tools, (4) removing of cerebellums and brain stems of the baseline images using inhouse tools, and (5) masking of the brains of the follow-up images using the same brain mask of their corresponding baseline image. The motivation of rigid registration of all longitudinal images to an atlas space in step (2) is as follows. Direct registration of the follow-up images to the baseline image will inevitably introduce interpolation to the followup images but not the baseline image, which results in smoother follow-up images in contrast to the original baseline image (Yushkevich et al., 2010; Reuter and Fischl, 2011). Therefore, to remove the bias of interpolation, all longitudinal images are transformed onto the atlas space by one step transform. Note that in our method all linear registrations are performed by FLIRT (Jenkinson and Smith, 2001) in Oxford FSL tools; however, more sophisticated methods, such as a highly accurate inverse consistent method in Reuter et al. (2010), can also be adopted. 


\subsection{Groupwise registration and consistent tissue segmentation}

Considering the nonlinear longitudinal morphological changes of human brain MR images of a subject, instead of averaging rigidly-aligned images as did in the existing methods (Reuter and Fischl, 2011; Nakamura et al., 2011), a nonlinear groupwise registration method (Wu et al., 2011) is first adopted to obtain the unbiased group-mean image of longitudinal images, as well as the deformation fields from each longitudinal image to the group-mean image. Then, the group-mean image of the subject will be used to derive the initial cortical surfaces for all longitudinal images of the same subject.

Tissue segmentation is one of the most important steps towards cortical surface reconstruction. And longitudinally-inconsistent tissue segmentation will affect the longitudinal cortical surface reconstruction results seriously. To achieve longitudinallyconsistent tissue segmentation results, CLASSIC (Xue et al., 2006) is adopted to perform consistent tissue segmentation on longitudinal images, which iteratively performs joint segmentation of longitudinal images and refinement of longitudinal deformations using a 4D elastic warping (Shen and Davatzikos 2002, 2004). To deal with the tissue segmentation errors in deep and narrow sulcal regions due to the imaging noises and partial volume effects, which often lead to inaccurate estimation of the central and outer cortical surfaces (Han et al., 2004; Kim et al., 2005), we use the anatomically consistent enhancement (ACE) method in Han et al. (2004) to generate a no-more-than-one-voxel thick separation between opposite sulcal GM banks for recovering deep buried sulci by modifying the initial segmented GM volume.

\subsection{Cortical surface reconstruction from group-mean image}

Reconstruction of cortical surfaces from the group-mean image could be considered as a problem of cortical surfaces reconstruction from a single MR image. In our method, the inner cortical surface is first reconstructed, and then the inner cortical surface is deformed under imposed internal and external forces to reconstruct both the central and outer cortical surfaces. This strategy has been extensively used for cortical surface reconstruction from a single image in the literature (Xu et al., 1999; Dale et al., 1999; Han et al., 2004; Kim et al., 2005; Liu et al., 2008), since the inner cortical surface is relatively easier to be identified than the central and outer cortical surfaces. Topological correctness is always an important issue in cortical surface reconstruction (Mangin et al., 1995; Fischl et al., 2001; Shattuck and Leahy, 2001; Kriegeskorte and Goebel, 2001; Han et al., 2002; Ségonne et al., 2007). To obtain the topologically-correct inner cortical surface, the topology of the WM volume is first corrected by a graph based method in Shattuck and Leahy (2001) as implemented in BrainSuite (Shattuck and Leahy, 2002) to ensure a spherical topology, and then the Marching cubes method (Lorensen and Cline, 1987) is used to convert the boundary of the topology-corrected WM volume into an explicit cortical surface representation. Using a deformable surface method, this reconstructed rough inner cortical surface is deformed under imposed internal and external forces to obtain the refined inner cortical surface, as well as to achieve the reconstruction of the central and outer cortical surfaces one-by-one. Since the deformable surface for cortical surface reconstruction from a single image can be considered as a special case of longitudinal cortical surface reconstruction, the general deformable surface will be detailed in Section 2.4. Fig. 2 shows an example of the reconstructed inner, central, and outer cortical surfaces of a group-mean image by our method.

\subsection{Longitudinal cortical surface reconstruction}

The reconstructed cortical surfaces of the group-mean image are warped to each longitudinal image using the corresponding deformation field generated by the groupwise registration method (Wu et al., 2011), and further jointly deformed for cortical surface reconstruction of 
longitudinal images using a deformable surface method (Kass et al., 1987), as popularly used in the literature for cortical surface reconstruction (Davatzikos and Bryan, 1996; Xu et al., 1999; Dale et al., 1999; Kim et al., 2005; Eskildsen and Ostergaard, 2006; Liu et al., 2008). Since the warped cortical surfaces are generally very close to the target surfaces (inner, central and outer cortical surfaces), they just need to be refined. For longitudinal surface reconstruction with $n$ time points, the deformable surfaces at 4D (3D spatial $+1 \mathrm{D}$ temporal) domain are parameterized as $\left\{\mathbf{x}_{t}(\mathbf{u})=\left[x_{t}(\mathbf{u}), y_{t}(\mathbf{u}), z_{t}(\mathbf{u})\right]^{T}, \mathbf{u}=\left(u_{1}, u_{2}\right) \in[0,1] \times\right.$ $[0,1], t \in\{1, \ldots, n\}\}$, which can be obtained by minimizing the following energy function:

$$
E=\sum_{t=1}^{n}\left(\int\left(\frac{1}{2}\left(\alpha \sum_{i=1}^{2}\left|\mathbf{x}_{t, i}\right|^{2}+\beta \sum_{i, j=1}^{2}\left|\mathbf{x}_{t, i j}\right|^{2}+\gamma\left|\mathbf{x}_{t}^{\prime}\right|^{2}\right)+E^{e x t}\left(\mathbf{x}_{t}\right)\right) d \mathbf{u}\right)
$$

where parameters $\alpha$ and $\beta$ control the tension and rigidity of surfaces, respectively. And the parameter $\gamma$ controls the temporal smoothness of surfaces. $\mathbf{x}_{t, i}$ and $\mathbf{x}_{t, i j}$ denote the first and the second partial derivative of $\mathbf{x}_{t}$ w.r.t. $\mathbf{u}_{i}$, respectively. And $\mathbf{x}_{t}^{\prime}$ denotes the finite difference of $\mathbf{x}_{t}$ w.r.t. time $t$. And $E^{e x t}\left(\mathbf{x}_{t}\right)$ is the external energy derived from the image at time $t$. The solution to the above energy minimization problem can be obtained by solving the following dynamic equation (Xu et al., 1999; Liu et al., 2008):

$$
\mathbf{x}_{t, \tau}=\mathbf{F}^{\text {int }}\left(\mathbf{x}_{t}\right)+\mathbf{F}^{\text {ext }}\left(\mathbf{x}_{t}\right)
$$

where the internal force is defined as $\mathbf{F}^{\text {int }}\left(\mathbf{x}_{t}\right)=\alpha \Delta_{\mathbf{u}} \mathbf{x}_{t}-\beta \Delta_{\mathbf{u}}\left(\Delta_{\mathbf{u}} \mathbf{x}_{t}\right)+\gamma \mathbf{x}_{t}^{\prime \prime}$ and the external force $\mathbf{F}^{e x t}\left(\mathbf{x}_{t}\right)$ will be defined later. Here, $\Delta_{\mathbf{u}}=\partial^{2} /\left(\partial u_{1}\right)^{2}+\partial^{2} /\left(\partial u_{2}\right)^{2}$ is the Laplacian operator to

$\mathbf{u}$. And $\mathbf{x}_{t}{ }^{\prime \prime}$ denotes the second order finite difference of $\mathbf{x}_{t}$ w.r.t. time $t$. In the internal force, the first two terms are the spatial regularizing forces, and the third term is the temporal regularizing force, which is used to impose temporal constraint along the time $t$. Note that the deformable surface is treated as a function of surface evolution time $\tau$. If only one time point exists, the temporal regularizing force will be 0 and the above deformable surface can be used for cortical surface reconstruction from the group-mean image as mentioned above.

The external force plays a central role to drive the initial surfaces towards the target surfaces. For cortical surface reconstruction, the general external force is designed as:

$$
\mathbf{F}^{\text {ext }}\left(\mathbf{x}_{t}\right)=G\left(\mathbf{x}_{t}\right) \cdot \mathbf{F}^{G M}\left(\mathbf{x}_{t}\right)+\left(1-G\left(\mathbf{x}_{t}\right)\right) \cdot \mathbf{F}^{\operatorname{Non} G M}\left(\mathbf{x}_{t}\right)
$$

where $G\left(\mathbf{x}_{t}\right)$ is the GM indicator function and defined as: if $\mathbf{x}_{t}$ is in GM, $G\left(\mathbf{x}_{t}\right)=1$; otherwise, $G\left(\mathbf{x}_{t}\right)=0 . \mathbf{F}^{G M}\left(\mathbf{x}_{t}\right)$ is a force activated inside of GM and will be defined later. And $\mathbf{F}^{\operatorname{NonGM}}\left(\mathbf{x}_{t}\right)$ is a force activated outside of GM and defined as:

$$
\mathbf{F}^{N o n G M}\left(\mathbf{x}_{t}\right)=D\left(\mathbf{x}_{t}\right) \cdot\left(2 W\left(\mathbf{x}_{t}\right)-1\right) \cdot \mathbf{n}\left(\mathbf{x}_{t}\right)
$$

where $W\left(\mathbf{x}_{t}\right)$ is the WM indicator function, and $\mathbf{n}\left(\mathbf{x}_{t}\right)$ is the outward-oriented unit normal vector. $D\left(\mathbf{x}_{t}\right)$ is the force strength at vertex $\mathbf{x}_{t}$. For inner and outer surface reconstruction, $D\left(\mathbf{x}_{t}\right)$ is the distance along the direction of $\left(2 W\left(\mathbf{x}_{t}\right)-1\right) \mathbf{n}\left(\mathbf{x}_{t}\right)$ to the WM/GM and GM/CSF interfaces, respectively. For central surface reconstruction, $D\left(\mathbf{x}_{t}\right)$ is set as the average distance along the direction of $\left(2 W\left(\mathbf{x}_{t}\right)-1\right) \mathbf{n}\left(\mathbf{x}_{t}\right)$ to WM/GM and GM/CSF interfaces.

The force $\mathbf{F}^{G M}\left(\mathbf{x}_{t}\right)$ is derived from the Laplace's equation (Jones et al., 2000) of the GM layer, which has been widely used for computing cortical thickness in brain MR images. Mathematically, Laplace's equation is a second-order partial differential equation for a scalar field $\phi$ enclosed between two interfaces: 


$$
\Delta \phi=\frac{\partial^{2} \phi}{\partial x^{2}}+\frac{\partial^{2} \phi}{\partial y^{2}}+\frac{\partial^{2} \phi}{\partial z^{2}}=0
$$

The solutions of Laplace's equation are harmonic functions (Jones et al., 2000). By setting the WM as the minimal value and the CSF as the maximum value, the Laplace's equation is solved inside of the GM to obtain the harmonic function. The normalized gradient vector field of the harmonic function and the streamlines to both WM/GM and GM/CSF interfaces are computed for each point in the GM. One important advantage of the Laplace's equation is that it establishes a one-to-one correspondence between WM/GM and GM/CSF interfaces and the streamlines of the harmonic function can never intersect each other. This elegant property helps preserve the topology of the deformable surface in GM when defining the force $\mathbf{F}^{G M}\left(\mathbf{x}_{t}\right)$ using the gradient vector field of the harmonic function. Denote the normalized gradient vector field of the harmonic function as $\mathbf{T}\left(\mathbf{x}_{t}\right)$. And also denote the lengths of streamlines from a point $\mathbf{x}_{t}$ in GM to WM/GM and GM/CSF interfaces as $L_{0}\left(\mathbf{x}_{t}\right)$ and $L_{1}\left(\mathbf{x}_{t}\right)$, respectively. Thus, the force $\mathbf{F}^{G M}\left(\mathbf{x}_{t}\right)$ for inner, central and outer cortical surfaces reconstruction can be respectively defined as:

$$
\begin{gathered}
\mathbf{F}_{\text {inner }}^{G M}\left(\mathbf{x}_{t}\right)=-\mathbf{T}\left(\mathbf{x}_{t}\right) \cdot L_{0}\left(\mathbf{x}_{t}\right) \\
\mathbf{F}_{\text {central }}^{G M}\left(\mathbf{x}_{t}\right)=\mathbf{T}\left(\mathbf{x}_{t}\right) \cdot\left(L_{1}\left(\mathbf{x}_{t}\right)-L_{0}\left(\mathbf{x}_{t}\right)\right) \\
\mathbf{F}_{\text {outer }}^{G M}\left(\mathbf{x}_{t}\right)=\mathbf{T}\left(\mathbf{x}_{t}\right) \cdot L_{1}\left(\mathbf{x}_{t}\right)
\end{gathered}
$$

The central idea behind this setting is that the direction of $\mathbf{F}^{G M}\left(\mathbf{x}_{t}\right)$ should always point toward the target surface and the magnitude of $\mathbf{F}^{G M}\left(\mathbf{x}_{t}\right)$ should be directly proportional to the distance to the target surface. Fig. 3 (a) and (b) illustrate the streamlines to GM/CSF interfaces in GM where deep sulci have been recovered. And Fig. 3 (c) and (d) illustrate the force directions for central and outer surface reconstruction in GM, respectively.

To preserve the topology when deforming surfaces, which is of great importance for generating topologically correct cortical surfaces, a fast triangle-triangle intersection detection method (Moller, 1997) is used, similar to the method in Dale et al. (1999). Once self-intersection is detected when deforming a vertex, the deformation is reduced until to a location where self-intersection no longer exists. Fig. 4 shows an example of reconstructed outer cortical surfaces of longitudinal images of a subject with 8 time points color-coded by cortical thickness, which is calculated as the closet distance between the inner and outer cortical surfaces (Fischl and Dale, 2000). Fig. 5 shows an example of the reconstructed longitudinal inner, central and outer cortical surfaces around the central sulcus in Fig. 4 overlaid in the corresponding 2D image slices. As we can see from Fig. 4, the cortical thickness is relatively thin around the central sulcus, and such observation is consistent with the findings in other surface reconstruction methods (Zeng et al., 1999; MacDonald et al., 2000; Han et al., 2004). We can also observe the overall decline trend of the cortical thickness in aging, which is consistent to the findings in the literature (Resnick et al., 2000, 2003; Salat et al., 2004; Rettmann et al., 2006; Fjell et al., 2009a, 2009b). 


\section{Results}

In this section, a set of experiments are conducted to evaluate our proposed longitudinal cortical surface reconstruction method. Currently, all the parameters in our method are set experimentally. And the parameters in the deformable surface are set as: $\alpha=0.25, \gamma=0.1$, and further fixed for all experiments. The parameter $\beta$, which controls the rigidity of the deformable surface, is set as 0 as suggested by Xu et al. 1999 and Liu et al. 2008. With the above parameter setting, reasonable cortical surface reconstruction results have been obtained for longitudinal images as will be reported below. In the experiments, we also found that the results are not sensitive to small changes of parameters.

\subsection{Results on ADNI dataset}

Data used in the preparation of this section were obtained from the Alzheimer's Disease Neuroimaging Initiative (ADNI) database (adni.loni.ucla.edu). The ADNI was launched in 2003 by the National Institute on Aging (NIA), the National Institute of Biomedical Imaging and Bioengineering (NIBIB), the Food and Drug Administration (FDA), private pharmaceutical companies and non-profit organizations, as a $\$ 60$ million, 5-year publicprivate partnership. The primary goal of ADNI has been to test whether serial magnetic resonance imaging (MRI), positron emission tomography (PET), other biological markers, and the progression of mild cognitive impairment (MCI) and early Alzheimer's disease (AD). Determination of sensitive and specific markers of very early AD progression is intended to aid researchers and clinicians to develop new treatments and monitor their effectiveness, as well as lessen the time and cost of clinical trials. ADNI is the result of efforts of many coinvestigators from a broad range of academic institutions and private corporations, and subjects have been recruited from over 50 sites across the U.S. and Canada. The initial goal of ADNI was to recruit 800 adults, ages 55 to 90, to participate in the research - approximately 200 cognitively normal older individuals to be followed for 3 years, 400 people with MCI to be followed for 3 years and 200 people with early AD to be followed for 2 years.

To validate the proposed method, we applied the method to both healthy and diseased subject groups. Specifically, four groups of subjects in ADNI are adopted, including the normal control (NC), stable mild cognitive impairment (S-MCI), progressive mild cognitive impairment (P-MCI), and Alzheimer's Disease (AD). The S-MCI subjects are the subjects that do not convert to AD even after several years of follow-up, while the P-MCI subjects are the subjects that finally developed probable AD. In all 4 groups, each subject has been scanned at least four times. The scan intervals between 1-2 and between 2-3 time points are around 6 months, and the scan interval between 3-4 time points are around 12 months. The demographic and clinical information of all selected subjects in the four groups are provided in Table 1. For more details of the criterion of selection of these subjects, we refer to Li et al. 2011.

3.1.1 Validation of Accuracy-To evaluate the accuracy of the longitudinal inner and outer cortical surface reconstruction results, we compare the GM volume in the tissuesegmented image (denote as A) with the GM volume enclosed by the corresponding reconstructed inner and outer surfaces (denote as B) as did in Lee et al. (2006). Three statistical values are calculated, including: (1) true positive: $(A \cap \bar{B}) / A$, (2) false negative: $(A$ $\cap \bar{B} / A$, (3) false positive: $(\bar{A} \cap B) / A$, similar to the measurements adopted in Lee et al. (2006). To evaluate the accuracy of the longitudinal central cortical surface reconstruction results, we calculate the percentage of vertices of the reconstructed central surface falling outside the GM (non-GM vertices), as adopted in Liu et al. (2008), since the central cortical surface is converged to the inside of GM. The proposed method is applied to 10 randomly 
selected normal healthy subjects, each with 4 time points. The average true positive, false negative, false positive, and percentage of non-GM vertices of 4 time points for each subject are shown in Fig. 6. For inner and outer cortical surfaces, the average true positive, false negative and false positive of our method are around 0.74, 0.26 and 0.15, respectively, indicating the relative accuracy of the proposed method. For central cortical surfaces, the average percentage of non-GM vertices of the reconstructed central surfaces by our method is around 0.03, showing the good performance of the proposed method. The above measurements are further compared in Fig. 6 to the results from the longitudinal processing pipeline in FreeSurfer, indicating that our method obtains better results. However, it is worth noting that, since FreeSurfer uses different tissue segmentation method from ours (4D-tissue segmentation), the improvement by our method may be also contributed from the tissue segmentation results.

To further validate the accuracy of the reconstructed inner and outer cortical surfaces, we simulate brain images from our reconstructed inner and outer cortical surfaces using the method in Lee et al. (2006). Briefly, the voxels inside the inner cortical surface are labeled as WM, and the voxels between the inner and outer cortical surfaces are labeled as GM, while the voxels between the skull and the outer cortical surface are labeled as CSF. Thus, the longitudinal image sequences are simulated from the corresponding reconstructed inner and outer cortical surfaces. Then the inner and outer cortical surfaces reconstructed from the simulated longitudinal images are compared to those original inner and outer cortical surfaces, which are treated as the "ground truth". The average surface distance errors between the two sets of surfaces are calculated and further averaged for 4 time points of each subject. The distance errors of the inner and outer cortical surfaces of 10 simulated subjects are shown in Fig. 7. On average, the average distance errors of the inner and outer cortical surfaces are all around $0.6 \mathrm{~mm}$, indicating the relative accuracy of our proposed method. To further validate the accuracy of the central cortical surfaces, we also simulate brain images from our reconstructed central surfaces using the method in Liu et al. (2008). Specifically, a thickness value following a Gaussian distribution with the mean value $3.0 \mathrm{~mm}$ and variance $1.0 \mathrm{~mm}$ is generated for each point of the central cortical surface by keeping the thickness locally smooth, and all voxels inside the half thickness range are labeled as GM. Also, all voxels enclosed by GM are defined as WM, and all the voxels between the skull and GM are labeled as CSF. Similarly, the reconstructed central cortical surfaces from the simulated longitudinal images are compared to those original central cortical surfaces. The distance errors of central cortical surfaces of 10 simulated subjects are also shown in Fig 7. The average distance errors of central cortical surfaces are around $0.55 \mathrm{~mm}$, indicating the relative accuracy of our proposed method.

3.1.2 Evaluation of Consistency-In this section, we compare the capabilities of consistently capturing longitudinally morphological cortical changes by three methods, including our method, our method without temporal constraint (i.e., by setting the parameter $\gamma=0$ ), and the longitudinal processing pipeline in FreeSurfer (Reuter and Fischl, 2011). We apply the three methods on 25 normal healthy subjects in the ADNI dataset to reconstruct longitudinal cortical surfaces and calculate the cortical thicknesses as defined in Fischl and Dale (2000), since one fundamental application of cortical surface reconstruction is to measure the cortical thickness. We first parcellate cortical surfaces into 78 ROIs (Regions Of Interest) using an atlas-based brain warping method (Shen and Davatzikos, 2002; Liu et al., 2004) based on the AAL atlas (Tzourio-Mazoyer et al., 2002) and then merge these ROIs into four lobes on each hemisphere, including frontal lobe, parietal lobe, temporal lobe and occipital lobe, in order to evaluate the longitudinal consistency of the average cortical thickness in each lobe. For quantitative comparison, a linear regression is performed on the longitudinal trajectories of average thickness of each lobe in left hemisphere of each subject, and the residuals are calculated. Smaller residual indicates that the longitudinal trajectory of 
cortical thicknesses is smoother. Since the longitudinal change of cortical thickness in short time (such as 6 months) for normal aging brains is generally very subtle, it is naturally expected that the average cortical thickness in each lobe should change smoothly along the time. The average residuals by our method in left frontal, parietal, temporal and occipital lobes are $0.0033 \pm 0.0027 \mathrm{~mm}, 0.0035 \pm 0.0023 \mathrm{~mm}, 0.0054 \pm 0.0033 \mathrm{~mm}$ and $0.004 \pm$ $0.0027 \mathrm{~mm}$, respectively. And the average residuals by our method without temporal constraint in left frontal, parietal, temporal and occipital lobes are $0.0174 \pm 0.0121 \mathrm{~mm}$, $0.0184 \pm 0.0133 \mathrm{~mm}, 0.0225 \pm 0.0162 \mathrm{~mm}$ and $0.0146 \pm 0.0095 \mathrm{~mm}$, respectively. While the average residuals by FreeSurfer in left frontal, parietal, temporal and occipital lobes are $0.0331 \pm 0.0189 \mathrm{~mm}, 0.0358 \pm 0.0171 \mathrm{~mm}, 0.0298 \pm 0.0201 \mathrm{~mm}$ and $0.0298 \pm 0.0267 \mathrm{~mm}$, respectively. It can be concluded that the average residuals in each lobe by our method are much smaller than the other two methods, indicating the necessity and benefit of imposing temporal constraint for longitudinal cortical surface reconstruction.

To further demonstrate the consistency of our proposed method on each vertex, we compare the trajectories of positions of vertices on the reconstructed longitudinal outer cortical surface by our method with those by our method without temporal constraint and the longitudinal processing pipeline in FreeSurfer, as shown in Fig. 8 (a), (b) and (c). Clearly, the trajectories of positions of vertices by our method are much smoother than the other two methods, which contain many zigzag trajectories since no temporal constraint is incorporated into the longitudinal surface reconstruction procedure of other two methods. For quantitative evaluation, we also perform linear regression on the trajectories of positions of vertices on longitudinal outer cortical surfaces and calculate their residuals. Fig. 9 (a), (b) and (c) show the comparison of the residuals of linear regression of positions of vertices on longitudinal outer cortical surfaces of a subject by our method, our method without temporal constraint, and FreeSurfer, respectively. Fig. 9 (d) shows the comparison of the average residuals on longitudinal outer cortical surfaces of 10 randomly selected subjects by the three methods. As we can see, the residuals by our method are consistently smaller than those by other two methods. All these results demonstrate that the trajectories of positions of vertices on longitudinal outer cortical surfaces by our method are much more consistent and smoother.

To demonstrate the consistency of calculated cortical thicknesses on each vertex, we also compare the residuals of linear regression of cortical thickness of vertices on reconstructed longitudinal outer surface by our method with other two methods. Fig. 10 (a), (b) and (c) show the results on a subject by our method, our method without temporal constraint, and FreeSurfer, respectively. While Fig. 10 (d) shows the average residuals on the abovementioned 10 subjects by the three methods. As we can see, the residuals by our method are consistently smaller than those by other two methods, indicating the consistency of our reconstructed longitudinal cortical surfaces.

3.1.3 Application to Different Clinical Groups-To show the potential clinical applications of the proposed method, we applied the method for reconstruction of longitudinal cortical surfaces on four groups of subjects, including the NC, S-MCI, P-MCI and $\mathrm{AD}$ groups, in which each group contains about 40 subjects with 4 time points in 24 months. The demographic and clinical information of all subjects in the four groups are provided in Table 1 . All cortical surfaces were parcellated into gyrus-based ROIs using the above mentioned method. The longitudinal trajectoires of average cortical thicknesses of four lobes on the left hemipsheres and the whole brains of four groups of subjects across 4 time points are shown in Fig. 11. As we can see, both the baseline cortical thicknesses and the longitudinal thickness trajectoires are quite different among the four groups across different lobes. In all four groups at the baseline, the frontal and temporal lobes have relatively large cortical thickness, while the parietal and occipital lobes have relatively small 
cortical thickness. And in all four groups, the decline trend of cortical thickness is apparent in the frontal, parietal and temporal lobes, but not apparent in the occipital lobes. And the largest decreasing trend of cortical thickness is shown in the temporal lobes of the AD group, consistent to the findings in the literature (Holland et al. 2009; Fjell et al., 2009b). Overall, the NC group has the largest average cortical thickness at the baseline and also has the slowest longitudincal thickness decline trend. While the AD group has the smallest average cortical thickness at the baseline and the fastest longitudincal thickness decline trend. The trajectoires of longitudinal cortical thciknesses of S-MCI and P-MCI groups are in between that of the $\mathrm{NC}$ and $\mathrm{AD}$ groups. And the S-MCI group is relatively close to the $\mathrm{NC}$ group, and the P-MCI group is relatively close to the AD group, in terms of both the baseline thickness and longitudinal thickness decline trend.

To further inspect the longitudinal cortical thickness changes in smaller ROIs, Fig. 12 shows the average cortical thicknesses changes between 1-2,1-3 and 1-4 time points in the gyrusbased ROIs of the four groups. Here, each ROI is color-coded by the average cortical thickness change of the ROI. From Fig. 12, we can observe the overall longitudinally smooth and consistent decline trend of cortical thickness in most ROIs in all groups. As we can also see, in the same group, there exist quite different cortical thickness changes across different gyri. And across different groups, we can also observe quite different cortical thickness changes at the same gyrus. In general, in all four groups, the AD group shows the largest thickness decline, especially at the temporal lobes of both hemispheres, consistent to the findings in literature (Holland et al. 2009; Fjell et al., 2009b). Interestingly, in all 4 groups, the cortical thickness change of the postcentral gyrus is relatively small, consistent to the findings in Holland et al. 2009. The statistical significance of the ROI-based cortical thickness thinning is measured by the $\mathrm{p}$-value in paired $\mathrm{t}$-test with the null hypothesis that the endline thicknesses are smaller than the corresponding baseline thicknesses in each ROI. Fig. 13 shows the results of the four groups, respectively. In all 4 lobes, the temporal lobe shows the most significant thinning of the cortical thickness in all four groups. And among all four groups, the $\mathrm{AD}$ group shows the most significant thinning in most ROIs.

To test the capability of our method in distinguishing between different clinical groups, such as NC vs. AD, and S-MCI vs. P-MCI, the statistical significance of the ROI-based cortical thickness difference between different groups is measured by the p-value in unpaired $t$-test, and further compared with FreeSurfer. Specifically, for NC vs. AD, the null hypothesis is that the cortical thicknesses in AD are smaller than that of NC in each ROI. And for S-MCI vs. P-MCI, the null hypothesis is that the cortical thicknesses in P-MCI are smaller than that of S-MCI in each ROI. Note that the t-test is performed at both the baseline and endline.

Since the cortical thinning is larger in AD than NC and also larger in P-MCI than S-MCI, it is expected that the difference between $\mathrm{AD}$ and $\mathrm{NC}$ and also the difference between P-MCI and S-MCI should be more significant at the endline than the baseline. Fig. 14 shows the pvalues of ROI-based cortical thickness difference between NC and AD groups by our method and FreeSurfer, respectively. As we can see, at the baseline, both methods show signicant difference in almost all ROIs, except precentral and postcentral gyri at both hemispheres. However, our result shows more significant difference between two groups than FreeSurfer. At the endline, in our method, the significance is further increased in most ROIs especially in the frontal lobes of both hemispheres. While, in FreeSurfer, although the significance is further increased in the frontal lobes at both hemispheres, the significance is decreased at both temporal lobes. Similarly, Fig. 15 shows the p-values of ROI-based cortical thickness difference between S-MCI and P-MCI groups by our method and FreeSurfer, respectively. Note that it is much more difficult to distinguish S-MCI vs. P-MCI than NC vs. AD. At the baseline, our method shows the significant group difference at the temporal lobe and supramarginal cortices of both hemispheres, while FreeSurfer shows the significant group difference at less number of regions such as left temporal lobe, left 
supramarginal cortice and right inferior temporal gyrus. At the endline, both methods show more significant group difference at the regions detected at the baseline. All these results demonstrate that our method is able to capture different longitudinal cortical thickness patterns in different clinical groups, and can identify more significant group difference than FreeSurfer.

\subsection{Results on BLSA dataset}

To further extensively validate our proposed method, we apply the method for cortical surface reconstruction of longitudinal brain MR images of 10 elderly subjects from the Baltimore Longitudinal Study of Aging (BLSA) dataset (Resnick et al., 2000). In the 10 subjects, each subject has been successively scanned 8 or 9 times, with the interval about 1 year, therefore, the longitudinal cortex changes are more apparently in the BLSA dataset than the ADNI dataset. With the reconstructed longitudinal cortical surfaces by our proposed method, we calculate the cortical thickness map of each time point for each subject. The longitudinal trajectories of the average cortical thicknesses of whole cortical surfaces of the 10 subjects with and without longitudinal constraints are shown in Fig. 16 (i) and (j), respectively. Since the longitudinal change of the cortical thickness in one year for the normal aging brains is generally subtle, it is expected that the average cortical thicknesses should change smoothly along the time. As we can see, the average cortical thickness declines smoothly along the time by our proposed method with temporal constraint, while its trajectory is very bumpy by our method without temporal constraint. Similarly, by performing linear regression on the longitudinal trajectory of average thickness of each subject, the average fitting residuals of our method with and without temporal constraint are $0.017 \pm 0.009 \mathrm{~mm}$ and $0.037 \pm 0.018 \mathrm{~mm}$, respectively, rendering the benefits of imposing temporal constraint for longitudinal cortical surface reconstruction.

To further validate the method, the cortical ROI-based longitudinal trajectories of cortical thickness are analyzed. Fig. 16 (a), (c), (e) and (g) show the trajectories of average cortical thicknesses of frontal, parietal, temporal and occipital lobes on the left hemispheres of the 10 subjects by our method with temporal constraint, along with Fig. 16 (b), (d), (f) and (h) by our method without temporal constraint. After performing linear regression on each longitudinal trajectory, the average residuals by our method with temporal constraint (or without temporal constraint) in the left frontal, parietal, temporal and occipital lobes are $0.0285 \pm 0.0135 \mathrm{~mm}(0.0578 \pm 0.0277 \mathrm{~mm}), 0.0226 \pm 0.0113 \mathrm{~mm}(0.0461 \pm 0.0191 \mathrm{~mm})$, $0.0261 \pm 0.0126 \mathrm{~mm}(0.0556 \pm 0.0270 \mathrm{~mm})$ and $0.0163 \pm 0.0091 \mathrm{~mm}(0.0375 \pm 0.0211$ $\mathrm{mm}$ ), respectively. Again, as we can see, the trajectory of average thickness of each lobe by our method with temporal constraint is much more consistent and smoother than that by our method without temporal constraint. Meanwhile, the overall trend of cortical thinning in each lobe in aging can be clearly observed by our method with temporal constraint. Again, similar to the findings in ADNI dataset, we can also observe that frontal and temporal lobes have relatively large average cortical thickness, while parietal and occipital lobes have relatively small average cortical thickness.

\section{Discussion and Conclusion}

In the literature, several methods have been proposed for cortical surface reconstruction from longitudinal human brain MR images, such as the longitudinal processing pipeline in FreeSurfer (Reuter and Fischl, 2011) and CLADA (Nakamura et al., 2011). In the longitudinal processing pipeline in FreeSurfer (Reuter and Fischl, 2011), the inner cortical surface of the group-mean image of the rigidly aligned or the median image of longitudinal images is first reconstructed by tessellating the WM volume and further refining the tessellation by a deformable surface (Dale et al., 1999), and then the corresponding outer cortical surface is reconstructed by deforming the inner cortical surface using the 
deformable surface, and at last, the inner and outer cortical surfaces are placed into the longitudinal images as the initialization and separately deformed to reconstruct the longitudinal inner and outer cortical surfaces using the deformable surface (Dale et al., 1999). In CLADA (Nakamura et al., 2011), the initial inner cortical surface of the groupmean image of the rigidly aligned longitudinal images is first reconstructed by shrinkwarping an elliptic surface to the WM volume, and then the initial outer cortical surface is generated by deforming the inner cortical surface by a deformable surface (Nakamura et al., 2011), and next the initial inner and outer cortical surfaces are jointly deformed to obtain the final cortical surfaces of the group-mean image by the deformable surface, and at last, similar to FreeSurfer, the inner and outer cortical surfaces are placed into the longitudinal images as the initialization and separately deformed to reconstruct the longitudinal inner and outer cortical surfaces using the deformable surface (Nakamura et al., 2011). Although the above methods can guarantee that the reconstructed longitudinal cortical surfaces at different time points have the exactly same triangular mesh configuration and topology with the cortical surfaces of the group-mean image, no temporal constraint is imposed in these methods (Reuter and Fischl, 2011; Nakamura et al., 2011), thus the temporal trajectories of attributes (such as positions and cortical thicknesses) of vertices on longitudinal cortical surfaces are generally bumpy.

In this paper, we presented a deformable surface method for consistent reconstruction of cortical surfaces from longitudinal human brain MR images. In our method, the cortical surfaces of the group-mean image of all non-rigidly aligned longitudinal images are first reconstructed using a deformable surface method, and then the longitudinal cortical surfaces are reconstructed by using the cortical surfaces of the group-mean image as the initialization and meanwhile explicitly imposing temporal constraint in the deformable surface method. Experimental results demonstrate the necessity and benefit of imposing this temporal constraint. As we can see, our method can reveal very subtle morphological changes such as $0.02 \mathrm{~mm}$ in 24 months in normal aging. It should be noted that the proposed method is mainly developed for working on adult brain images with subtle and smooth longitudinal morphological changes across relatively uniform time points. Therefore, the method might not work well on subjects with dramatic longitudinal cortical changes at a time point or with highly uneven distributed time points. Note that since longitudinal smoothness is imposed in the method, the longitudinal changes, such as the maximum change of the cortical thickness, might be underestimated, however, as long as the assumption of smooth longitudinal change is effective (which should be true for normal aging and $\mathrm{AD}$ ), the proposed model will only slightly affect the range of variations. And also, a more conservative estimation of change is more preferable than an overestimation for longitudinal analysis (Reuter and Fischl, 2011). More importantly, it has been demonstrated the potential clinical application of the proposed method, since our method can reveal the difference of the longitudinal cortical thickness patterns among different groups. Recently, a voxel-based method for measuring longitudinal cortical thickness has been proposed (Li, 2010c), which, however, doesn't consider the inherent spherical topology of the cortex. Moreover, the longitudinal cortical surface reconstructions has much wider application than measuring only the cortical thickness achieved by the voxel-based method (Li, 2010c), e.g., studying and modeling cortical folding, cortical surface based registration, and functional mapping.

Although the proposed method for consistent reconstruction of longitudinal cortical surfaces has achieved reasonably good results, several procedures involved in our proposed method might be further improved. First, the linear alignment of longitudinal images is performed in our method by FLIRT (Jenkinson and Smith, 2001) in FSL tools currently. In the future, we will investigate whether the highly accurate inverse consistent method in Reuter et al. (2010) will further increase the accuracy of the linear alignment. Second, CLASSIC (Xue et al., 2006) is adopted for longitudinally consistent tissue segmentation to facilitate the 
longitudinal cortical surface reconstruction. And it is found that the longitudinal cortical surface reconstruction benefits from this method in contrast to independently performing tissue segmentation at each time point. However, since CLASSIC treats the baseline image different from the follow-up scans by using the baseline image as the reference for 4D registration, it might introduce some bias in this step. In the future, we will investigate unbiased longitudinal consistent segmentation by treating each longitudinal image equally. Third, the recovery of deep buried sulci is performed by the ACE method in Han et al. (2004) on each longitudinal image independently. In the future, we will investigate method on consistently recovering deep buried sulci in longitudinal images. At last, several parameters in the deformable surface are set experimentally currently, and the optimal parameter setting will be investigated in the future. Currently, our method takes about 2 hours for surface reconstruction from the mean image, and another 8 hours for longitudinal cortical surface reconstruction on 4 time points in ADNI subjects. In the future, we will investigate how to optimize the method. And after making the method more self-contained and stable, we intend to make it publicly available. Our future work will also include the further validation of our proposed method using more longitudinal data, and the analysis of longitudinal attribute changes of the reconstructed cortical surfaces for the aging and disease progression studies using cortical thickness, cortical area, and cortical folding.

\section{Acknowledgments}

The BLSA dataset used in this paper was provided by Dr. Susan Resnick and Dr. Christos Davatzikos. The authors would like to thank the anonymous reviewers for providing constructive suggestions that improved this paper.

Data collection and sharing for this project was funded by the Alzheimer's Disease Neuroimaging Initiative (ADNI) (National Institutes of Health Grant U01 AG024904). ADNI is funded by the National Institute on Aging, the National Institute of Biomedical Imaging and Bioengineering, and through generous contributions from the following: Abbott, AstraZeneca AB, Bayer Schering Pharma AG, Bristol-Myers Squibb, Eisai Global Clinical Development, Elan Corporation, Genentech, GE Healthcare, GlaxoSmithKline, Innogenetics, Johnson and Johnson, Eli Lilly and Co., Medpace, Inc., Merck and Co., Inc., Novartis AG, Pfizer Inc, F. Hoffman-La Roche, ScheringPlough, Synarc, Inc., as well as non-profit partners the Alzheimer's Association and Alzheimer's Drug Discovery Foundation, with participation from the U.S. Food and Drug Administration. Private sector contributions to ADNI are facilitated by the Foundation for the National Institutes of Health (www.fnih.org). The grantee organization is the Northern California Institute for Research and Education, and the study is coordinated by the Alzheimer's Disease Cooperative Study at the University of California, San Diego. ADNI data are disseminated by the Laboratory for Neuro Imaging at the University of California, Los Angeles. This research was also supported by NIH grants P30 AG010129, K01 AG030514, and the Dana Foundation.

\section{References}

Andrade A, Kherif F, Mangin JF, Worsley KJ, Paradis AL, Simon O, Dehaene S, Le Bihan D, Poline JB. Detection of fMRI activation using cortical surface mapping. Hum Brain Mapp. 2001; 12(2): 79-93. [PubMed: 11169872]

Cachia A, Mangin JF, Rivière D, Kherif F, Boddaert N, Andrade A, Papadopoulos-Orfanos D, Poline JB, Bloch I, Zilbovicius M, Sonigo P, Brunelle F, Régis J. A primal sketch of the cortex mean curvature: a morphogenesis based approach to study the variability of the folding patterns. IEEE Trans Med Imaging. 2003; 22(6):754-765. [PubMed: 12872951]

Dale AM, Fischl B, Sereno MI. Cortical surface-based analysis I: segmentation and surface reconstruction. Neuroimage. 1999; 9(2):179-194. [PubMed: 9931268]

Davatzikos C, Bryan N. Using a deformable surface model to obtain a shape representation of the cortex. IEEE Trans Med Imaging. 1996; 15(6):785-795. [PubMed: 18215958]

Eskildsen SF, Ostergaard LR. Active surface approach for extraction of the human cerebral cortex from MRI. Proc International Conference on Medical Image Computing and Computer Assisted Intervention 2006 vol. 2006; 2:823-830.

Fjell AM, Westlye LT, Amlien I, Espeseth T, Reinvang I, Raz N, Agartz I, Salat DH, Greve DN, Fischl B, Dale AM, Walhovd KB. High consistency of regional cortical thinning in aging across multiple samples. Cereb Cortex. 2009; 19(9):2001-2012. [PubMed: 19150922] 
Fjell AM, Walhovd KB, Fennema-Notestine C, McEvoy LK, Hagler DJ, Holland D, Brewer JB, Dale AM. One-year brain atrophy evident in healthy aging. J Neurosci. 2009; 29(48):15223-15231. [PubMed: 19955375]

Fischl B, Sereno MI, Dale AM. Cortical surface-based analysis. II: Inflation, flattening, and a surfacebased coordinate system. Neuroimage. 1999a; 9(2):195-207. [PubMed: 9931269]

Fischl B, Sereno MI, Tootell R, Dale AM. High-resolution intersubject averaging and a coordinate system for the cortical surface. Hum Brain Mapp. 1999b; 8(4):272-284. [PubMed: 10619420]

Fischl B, Dale AM. Measuring the thickness of the human cerebral cortex from Magnetic Resonance images. Proc Natl Acad Sci. 2000; 97(20):11050-11055. [PubMed: 10984517]

Fischl B, Liu A, Dale AM. Automated manifold surgery: constructing geometrically accurate and topologically correct models of the human cerebral cortex. IEEE Trans Med Imaging. 2001; 20(1): 70-80. [PubMed: 11293693]

Hagmann P, Cammoun L, Gigandet X, Meuli R, Honey CJ, Wedeen VJ, Sporns O. Mapping the structural core of human cerebral cortex. PLoS Biol. 2008; 6(7):e159. [PubMed: 18597554]

Han X, Xu C, Braga-Neto U, Prince JL. Topology correction in brain cortex segmentation using a multiscale, graph-based algorithm. IEEE Trans Med Imaging. 2002; 21(2):109-121. [PubMed: 11929099]

Han X, Pham DL, Tosun D, Rettmann ME, Xu C, Prince JL. CRUISE: cortical reconstruction using implicit surface evolution. Neuroimage. 2004; 23(3):997-1012. [PubMed: 15528100]

Han X, Jovicich J, Salat D, van der Kouwe A, Quinn B, Czanner S, Busa E, Pacheco J, Albert M, Killiany R, Maguire P, Rosas D, Makris N, Dale A, Dickerson B, Fischl B. Reliability of MRIderived measurements of human cerebral cortical thickness: the effects of field strength, scanner upgrade and manufacturer. Neuroimage. 2006; 32(1):180-194. [PubMed: 16651008]

Holland D, Brewer JB, Hagler DJ, Fenema-Notestine C, Dale AM. Subregional neuroanatomical change as a biomarker for Alzheimer's disease. Proc Natl Acad Sci USA. 2009; 106:20954 20959. [PubMed: 19996185]

Jenkinson M, Smith S. A global optimisation method for robust affine registration of brain images. Med Image Anal. 2001; 5(2):143-156. [PubMed: 11516708]

Jones SE, Buchbinder BR, Aharon I. Three-dimensional mapping of cortical thickness using Laplace's equation. Hum Brain Mapp. 2000; 11(1):12-32. [PubMed: 10997850]

Joshi M, Cui J, Doolittle K, Joshi S, Van Essen D, Wang L, Miller MI. Brain segmentation and the generation of cortical surfaces. Neuroimage. 1999; 9(5):461-476. [PubMed: 10329286]

Kass M, Witkin A, Terzopoulos D. Snakes: active contour models. Int J Comput Vis. 1987; 1(4):321331.

Kim JS, Singh V, Lee JK, Lerch J, Ad-Dab'bagh Y, MacDonald D, Lee JM, Kim SI, Evans AC. Automated 3-D extraction and evaluation of the inner and outer cortical surfaces using a Laplacian map and partial volume effect classification. Neuorimage. 2005; 27(1):210-221.

Kriegeskorte N, Goebel R. An efficient algorithm for topologically correct segmentation of the cortical sheet in anatomical MR volumes. Neuroimage. 2001; 14(2):329-346. [PubMed: 11467907]

Lee JK, Lee JM, Kim JS, Kim IY, Evans AC, Kim SI. A novel quantitative cross-validation of different cortical surface reconstruction algorithm using MRI phantom. Neuroimage. 2006; 31(2): 572-584. [PubMed: 16503170]

Li G, Guo L, Nie J, Liu T. Automatic cortical sulcal parcellation based on surface principal direction flow field tracking. Neuroimage. 2009; 46(4):923-937. [PubMed: 19328234]

Li G, Guo L, Nie J, Liu T. An automated pipeline for cortical sulcal fundi extraction. Med Image Anal. 2010a; 14(3):343-359. [PubMed: 20219410]

Li K, Guo L, Li G, Nie J, Faraco C, Cui G, Zhao Q, Miller LS, Liu T. Gyral folding pattern analysis via surface profiling. Neuroimage. 2010b; 52(4):1202-1214. [PubMed: 20472071]

Li Y, Wang Y, Xue Z, Shi F, Lin W, Shen D. ADNI. Consistent 4D cortical thickness measurement for longitudinal neuroimaging study. Proc International Conference on Medical Image Computing and Computer Assisted Intervention 2010 vol. 2010c; 2:133-142.

Li G, Shen D. Consistent sulcal parcellation of longitudinal cortical surfaces. Neuroimage. 2011; 57(1):76-88. [PubMed: 21473919] 
Li Y, Wang Y, Wu G, Shi F, Zhou L, Lin W, Shen D. ADNI. Discriminant analysis of longitudinal cortical thickness changes in Alzheimer's disease using dynamic and network features. Neurobiol Aging. 2011 In press.

Liu T, Shen D, Davatzikos C. Deformable registration of cortical structures via hybrid volumetric and surface warping. Neuroimage. 2004; 22(4):1790-17801. [PubMed: 15275935]

Liu T, Nie J, Tarokh A, Guo L, Wong ST. Reconstruction of central cortical surface from brain MRI image: method and application. Neuroimage. 2008; 40(3):991-1002. [PubMed: 18289879]

Lorensen WE, Cline HE. Marching cubes: A high- resolution 3-D surface construction algorithm. ACM Comput Graph. 1987; 21(4):163-170.

MacDonald D, Kabani N, Avis D, Evans AC. Automated 3-D extraction of inner and outer surfaces of cerebral cortex from MRI. Neuroimage. 2000; 12(3):340-356. [PubMed: 10944416]

Mangin JF, Frouin V, Bloch I, Régis J, Lopez-Krahe J. From 3D magnetic resonance images to structural representations of the cortex topography using topology preserving deformations. J Math Imaging Vision. 1995; 5(4):297-318.

Mangin J-F, Jouvent E, Cachia A. In-vivo measurement of cortical morphology: means and meanings. Curr Opin Neurol. 2010; 23(4):359-367. [PubMed: 20489617]

Moller T. A fast triangle - triangle intersection test. J Graphics Tools. 1997; 2(2):25-30.

Nakamura K, Fox R, Fisher E. CLADA: cortical longitudinal atrophy detection algorithm. Neuroimage. 2011; 54(1):278-289. [PubMed: 20674750]

Nie J, Guo L, Li G, Faraco C, Stephen Miller L, Liu T. A computational model of cerebral cortex folding. J Theor Biol. 2010; 264(2):467-478. [PubMed: 20167224]

Resnick S, Goldszal A, Davatzikos C, Golski S, Kraut M, Metter E, Bryan R, Zonderman A. One-year age changes in MRI brain volumes in older adults. Cereb Cortex. 2000; 10(5):464-472. [PubMed: 10847596]

Resnick SM, Pham DL, Kraut MA, Zonderman AB, Davatzikos C. Longitudinal magnetic resonance imaging studies of older adults: a shrinking brain. J Neurosci. 2003; 23(8):3295-3301. [PubMed: 12716936]

Rettmann ME, Kraut MA, Prince JL, Resnick SM. Cross-sectional and longitudinal analyses of anatomical sulcal changes associated with aging. Cereb Cortex. 2006; 16(11):1584-1594. [PubMed: 16400155]

Reuter M, Rosas HD, Fischl B. Highly accurate inverse consistent registration: a robust approach. Neuroimage. 2010; 53(4):1181-1196. [PubMed: 20637289]

Reuter M, Fischl B. Avoiding asymmetry-induced bias in longitudinal image processing. Neuroimage. 2011; 57(1):19-21. [PubMed: 21376812]

Salat DH, Buckner RL, Snyder AZ, Greve DN, Desikan RS, Busa E, Morris JC, Dale AM, Fischl B. Thinning of the cerebral cortex in aging. Cereb Cortex. 2004; 14(7):721-730. [PubMed: 15054051]

Schaer M, Cuadra MB, Tamarit L, Lazeyras F, Eliez S, Thiran JP. A surface-based approach to quantify local cortical gyrification. IEEE Trans Med Imaging. 2008; 27(2):161-170. [PubMed: 18334438]

Ségonne F, Pacheco J, Fischl B. Geometrically accurate topology-correction of cortical surfaces using nonseparating loops. IEEE Trans Med Imaging. 2007; 26(4):518-529. [PubMed: 17427739]

Shattuck DW, Leahy RM. Automated graph-based analysis and correction of cortical volume topology. IEEE Trans Med Imaging. 2001; 20(11):1167-1177. [PubMed: 11700742]

Shattuck DW, Leahy RM. BrainSuite: An automated cortical surface identification tool. Med Image Anal. 2002; 8(2):129-142. [PubMed: 12045000]

Shen D, Davatzikos C. HAMMER: hierarchical attribute matching mechanism for elastic registration. IEEE Trans Med Imaging. 2002; 21(11):1421-1439. [PubMed: 12575879]

Shen D, Davatzikos C. Measuring temporal morphological changes robustly in brain MR images via 4D template warping. Neuroimage. 2004; 21(4):1508-1517. [PubMed: 15050575]

Shi Y, Lai R, Toga AW. CoRPORATE: cortical reconstruction by pruning outliers with Reeb analysis and topology-preserving evolution. Inf Process Med Imaging 2011. 2011; 22:233-244. 
Sled JG, Zijdenbos AP, Evans AC. A nonparametric method for automatic correction of intensity nonuniformity in MRI data. IEEE Trans Med Imaging. 1998; 17(1):87-97. [PubMed: 9617910]

Smith SM. Fast robust automated brain extraction. Hum Brain Mapp. 2002; 17(3):143-155. [PubMed: 12391568]

Tao X, Prince JL, Davatzikos C. Using a statistical shape model to extract sulcal curves on the outer cortex of the human brain. IEEE Trans Med Imaging. 2002; 21(5):513-524. [PubMed: 12071622]

Thambisetty M, Wan J, Carass A, An Y, Prince JL, Resnick SM. Longitudinal changes in cortical thickness associated with normal aging. Neuroimage. 2010; 52(4):1215-1223. [PubMed: 20441796]

Thompson PM, Toga AW. A surface-based technique for warping 3-dimensional images of the brain. IEEE Trans Med Imaging. 1996; 15(4):402-417. [PubMed: 18215923]

Tzourio-Mazoyer N, Landeau B, Papathanassiou D, Crivello F, Etard O, Delcroix N, Mazoyer B, Joliot M. Automated anatomical labeling of activations in SPM using a macroscopic anatomical parcellation of the MNI MRI single-subject brain. Neuroimage. 2002; 15(1):273-289. [PubMed: 11771995]

Van Essen DC, Drury HA, Joshi S, Miller MI. Functional and structural mapping of human cerebral cortex: solutions are in the surfaces. Proc Natl Acad Sci. 1998; 95(3):788-795. [PubMed: 9448242]

Van Essen DC, Drury HA, Dickson J, Harwell J, Hanlon D, Anderson CH. An integrated software suite for surface-based analyses of cerebral cortex. J Am Med Inform Assoc. 2001; 8(5):443-459. [PubMed: 11522765]

Van Essen DC. Surface-based approaches to spatial localization and registration in primate cerebral cortex. Neuroimage. 2004; 23(Suppl 1):S97-107. [PubMed: 15501104]

Van Essen DC, Dierker DL. Surface-based and probabilistic atlases of primate cerebral cortex. Neuron. 2007; 56(2):209-225. [PubMed: 17964241]

von Economo, C. The Cytoarchitectonics of the Human Cerebral Cortex. Oxford Univ. Press; London: 1929.

Wu G, Jia H, Wang Q, Shen D. SharpMean: Groupwise registration guided by sharp mean image and tree-based registration. Neuroimage. 2011; 56(4):1968-1981. [PubMed: 21440646]

Xu C, Pham DL, Rettmann ME, Yu DN, Prince JL. Reconstruction of the human cerebral cortex from magnetic resonance images. IEEE Trans Med Imaging. 1999; 18(6):467-80. [PubMed: 10463126]

Xu M, Thompson PM, Toga AW. Adaptive reproducing kernel particle method for extraction of the cortical surface. IEEE Trans Med Imaging. 2006; 25(6):755-767. [PubMed: 16768240]

Xue Z, Shen D, Davatzikos C. CLASSIC: consistent longitudinal alignment and segmentation of serial image computing. Neuroimage. 2006; 30(2):388-399. [PubMed: 16275137]

Yushkevich PA, Avants BB, Das SR, Pluta J, Altinay M, Craige C. ADNI. Bias in estimation of hippocampal atrophy using deformation-based morphometry arises from asymmetric global normalization: an illustration in ADNI 3 T MRI data. Neuroimage. 2010; 50(2):434-445. [PubMed: 20005963]

Zeng X, Staib LH, Schultz RT, Duncan JS. Segmentation and measurement of the cortex from 3D MR images using coupled surfaces propagation. IEEE Trans Med Imaging. 1999; 18(10):100-111.

Zhang D, Guo L, Li G, Nie J, Jiang X, Deng F, Li K, Zhu D, Zhao Q, Liu T. Automatic cortical surface parcellation based on fiber density information. Proc IEEE International symposium on biomedical imaging. 2010; 2010:656-659.

Zilles, K. Cortex in the Human Nervous System. Academic Press; San Diego, CA: 1990. 


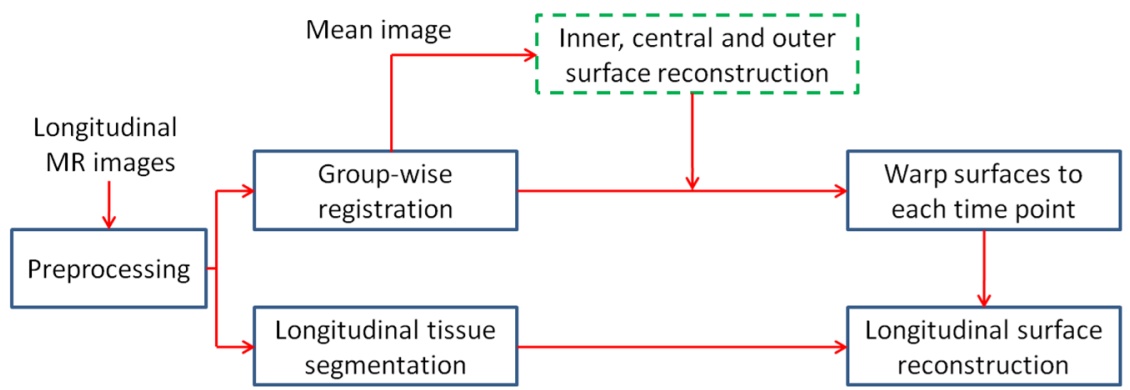

(a)

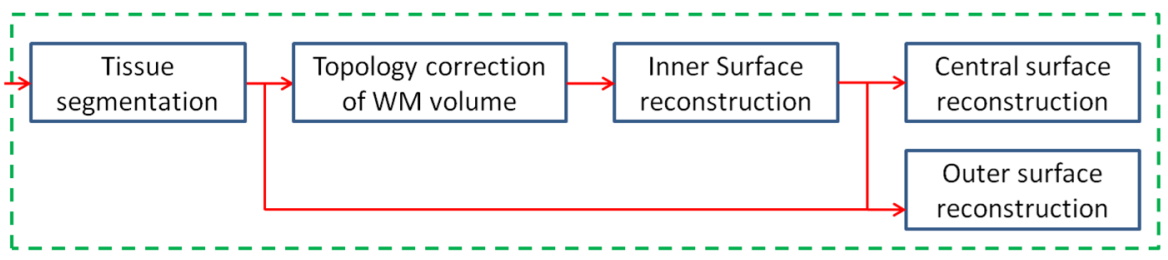

(b)

Fig. 1.

(a) The flow chart of the proposed longitudinal cortical surface reconstruction method. (b) The flow chart of the cortical surface reconstruction method from the group-mean image. 


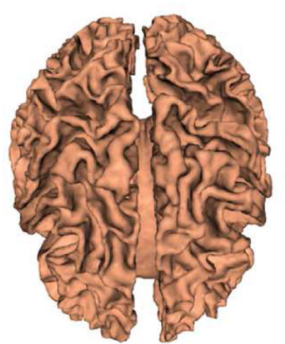

(a)

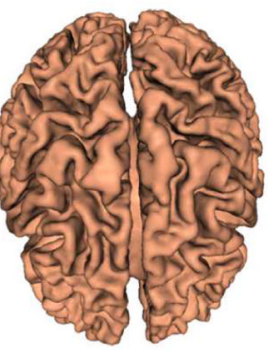

(b)

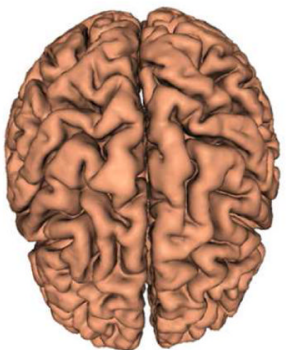

(c)

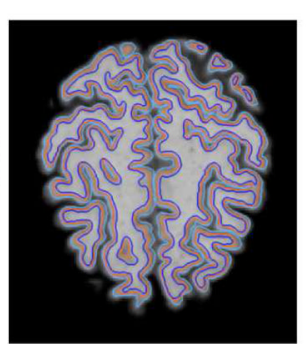

(d)

Fig. 2.

Reconstructed cortical surfaces from a group-mean image. (a) Inner surface. (b) Central surface. (c) Outer surface. (d) Three surfaces overlaid in a 2D image slice. Dark blue: inner; orange: central; light blue: outer. 


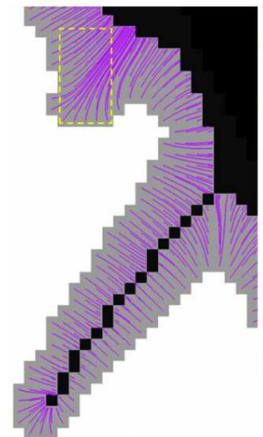

(a)

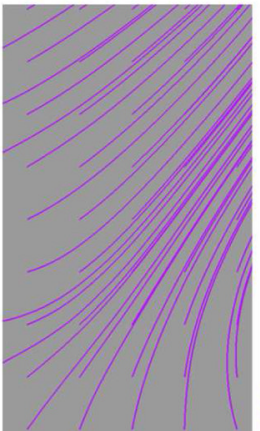

(b)

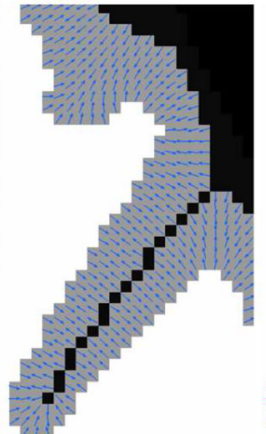

(c)

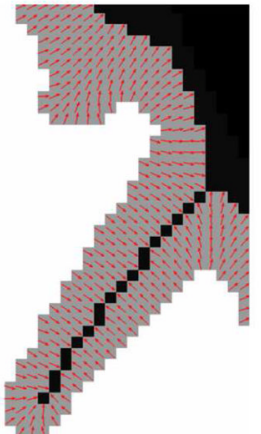

(d)

Fig. 3.

Illustration of streamlines and force directions in GM. (a) The streamlines to GM/CSF interface. (b) The zooming view of the yellow rectangular region in figure (a). (c) The force directions for central surface reconstruction. (d) The force directions for outer surface reconstruction. 


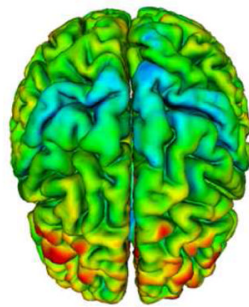

(a)

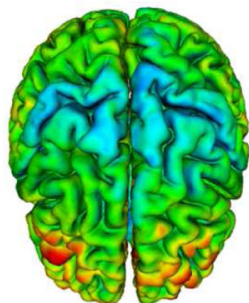

(e)

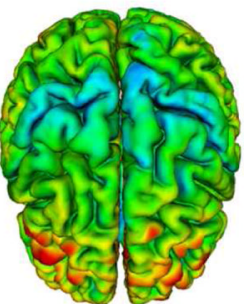

(b)

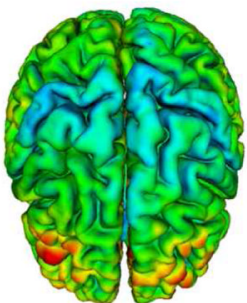

(f)

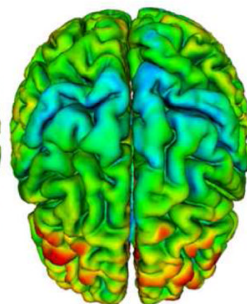

(c)

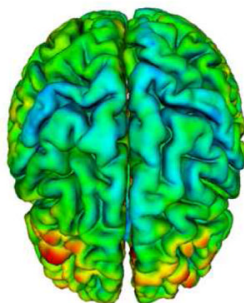

(g)

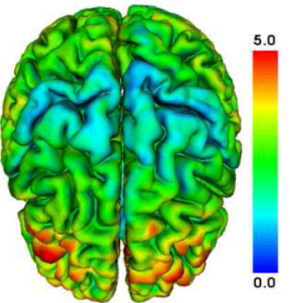

(d)

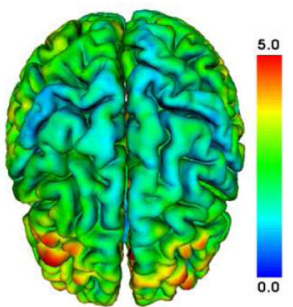

(h)

Fig. 4.

An example of reconstructed longitudinal outer cortical surfaces of a healthy subject with 8 time points (from figure (a) to figure (h)), color-coded by cortical thickness (mm). The color bars are shown on the right. 


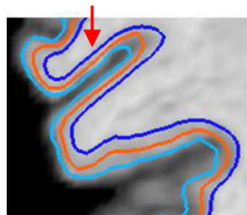

(a)

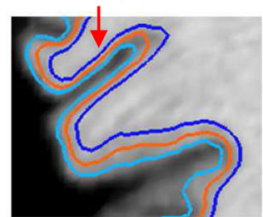

(e)

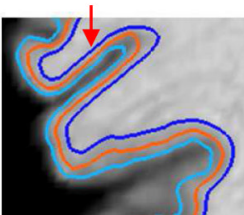

(b)

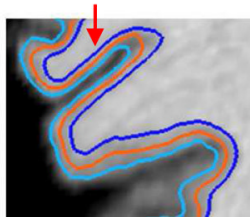

(c)

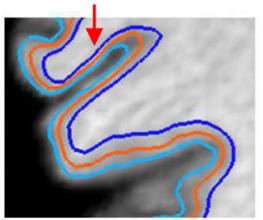

(d)

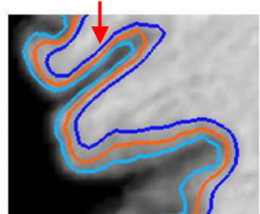

(f)

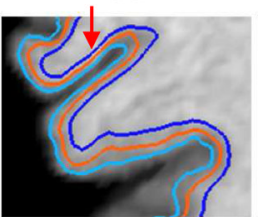

(g)

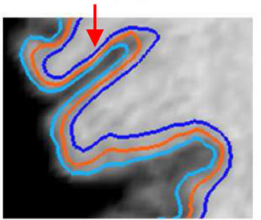

(h)

Fig. 5.

An example of the reconstructed longitudinal inner (blue curves), central (orange curves), and outer (light blue curves) cortical surfaces in Fig. 4 overlaid in the corresponding 2D image slices. The red arrows indicate the selected regions with cortical thinning. 


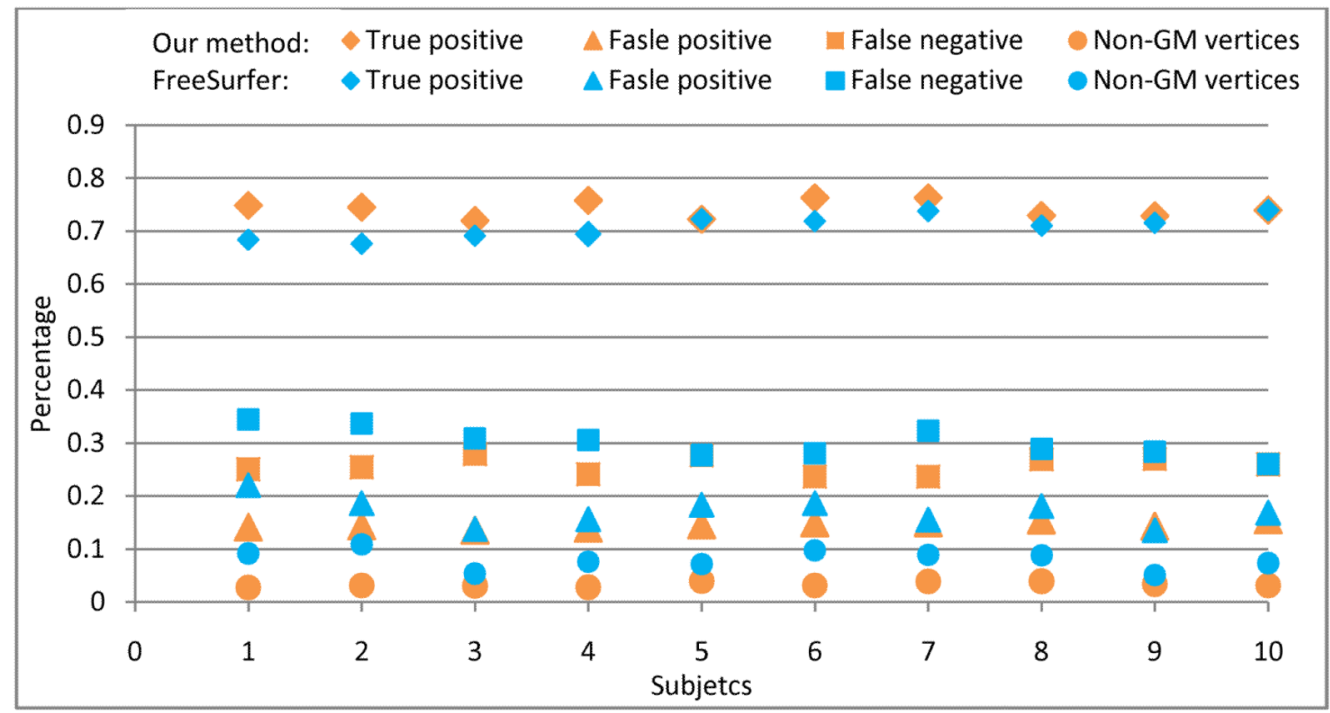

Fig. 6.

The average true positive, false negative, false positive, and percentage of non-GM vertices of 4 time points for each subject. Note that the true positive, false negative and false positive are used to validate the inner and outer cortical surfaces, and the percentage of non-GM vertices is used to validate the central cortical surfaces. Larger true positive indicates better results, while smaller false positive, false negative and non-GM vertices indicate better results. 


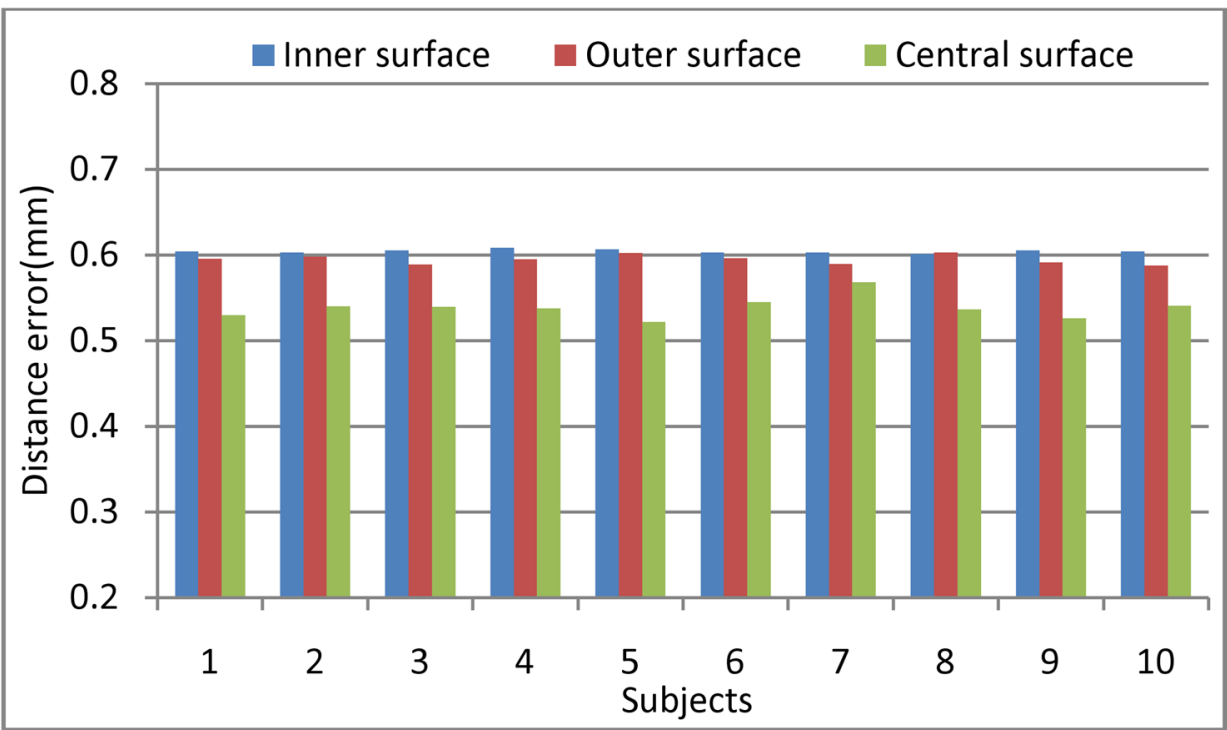

Fig. 7.

The average surface distance errors of the inner, central and outer cortical surfaces of 4 time points for each subject, compared to the "ground truth". 


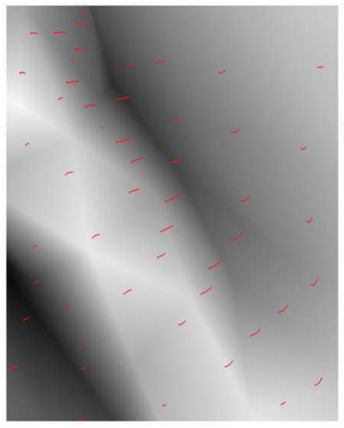

(a)

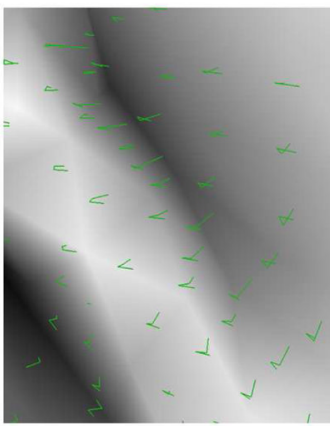

(b)

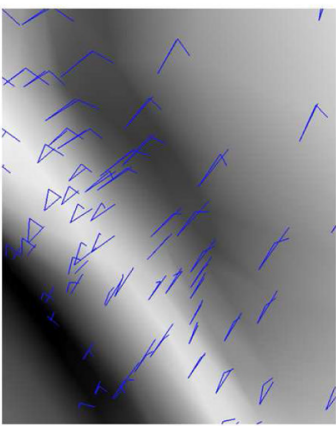

(c)

Fig. 8.

Comparison of the trajectories of positions of vertices on longitudinal outer cortical surfaces of a subject by our method (a), our method without temporal constraint (b), and the longitudinal processing pipeline in FreeSurfer (c). 


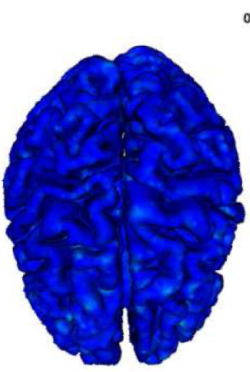

(a)

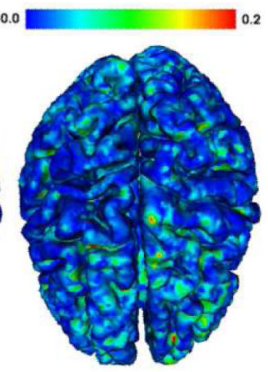

(b)

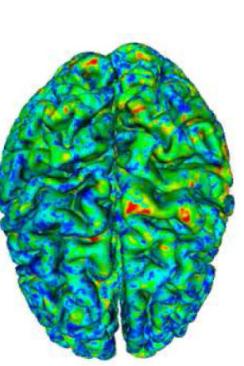

(c)

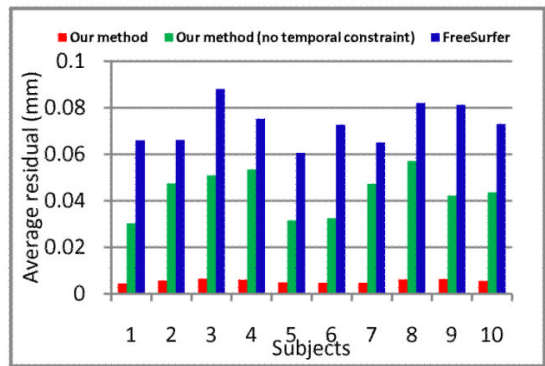

(d)

Fig. 9.

Comparison of the residuals of linear regression of trajectories of positions of vertices on longitudinal outer cortical surfaces by (a) our method, (b) our method without temporal constraint, and (c) FreeSurfer. (a), (b), (c): Color-coded residuals (mm) on a subject; (d) Average residuals on 10 subjects by the three methods. 


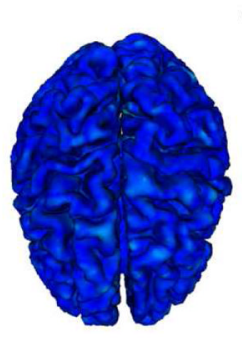

(a)

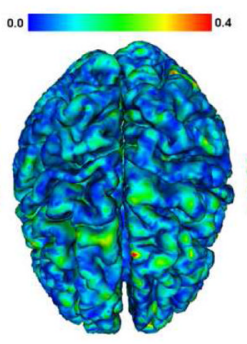

(b)

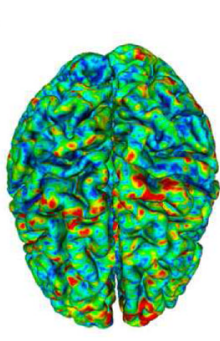

(c)

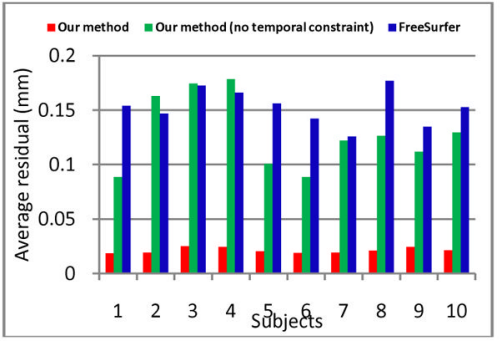

(d)

Fig. 10.

Comparison of the residuals of linear regression of trajectories of cortical thicknesses of vertices on longitudinal outer cortical surfaces by (a) our method, (b) our method without temporal constraint, and (c) FreeSurfer. (a), (b), (c): Color-coded residuals (mm) on a subject; (d) Average residuals on 10 subjects by the three methods. 


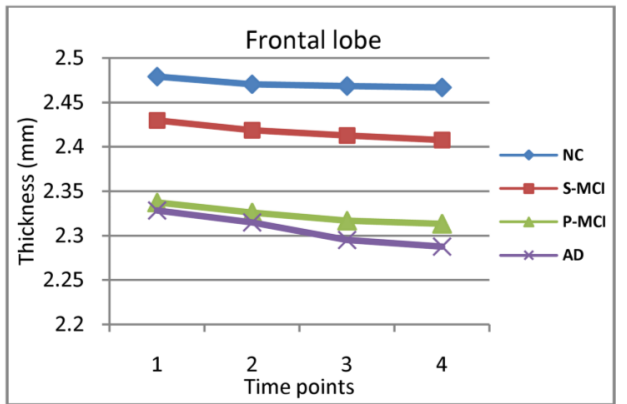

(a)

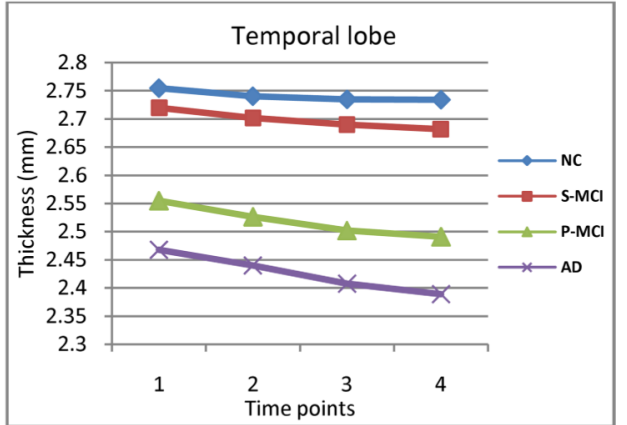

(c)

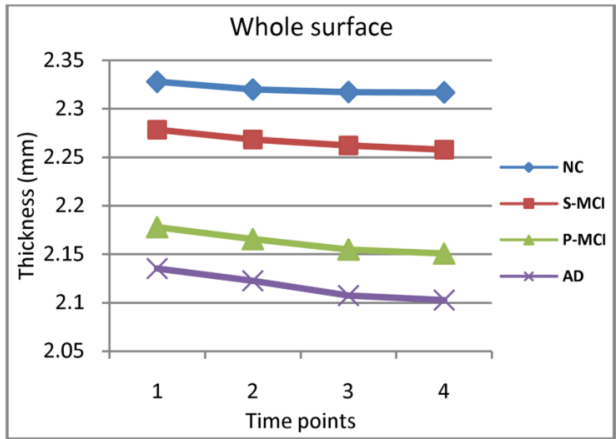

(e)

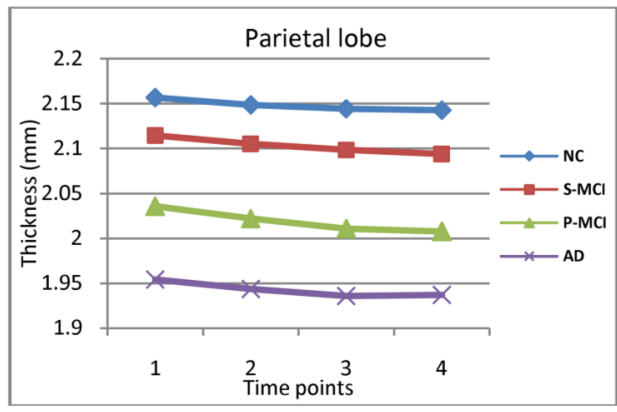

(b)

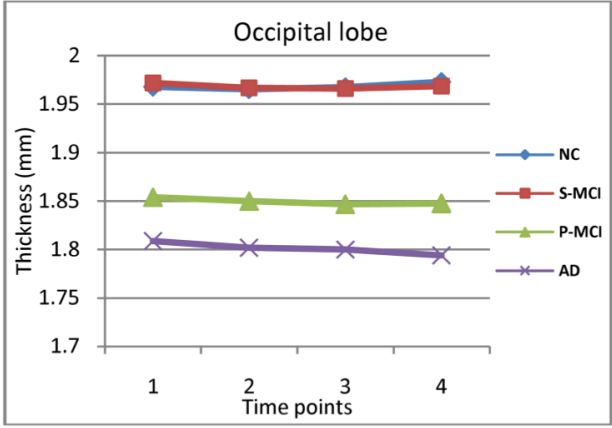

(d)

Fig. 11.

The longitudinal trajectoires of average cortical thicknesses of four lobes on the left hemipshere and the whole surface of four groups of subjects (NC, S-MCI, P-MCI and AD) across 4 time points. Figures (a), (b), (c) and (d) show the results on frontal, parietal, temporal and occipital lobes, respectively. Figure (e) shows the results on the whole surface. As we can see, the longitudinal cortical thickness patterns are quite different among 4 groups. 


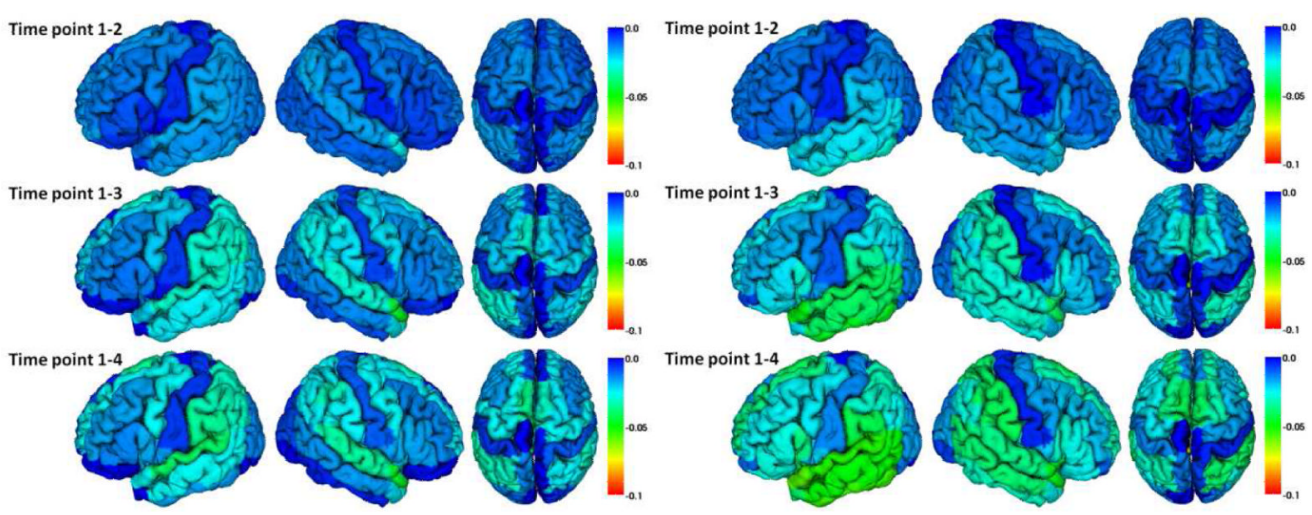

(a) $\mathrm{NC}$

(b) S-MCI

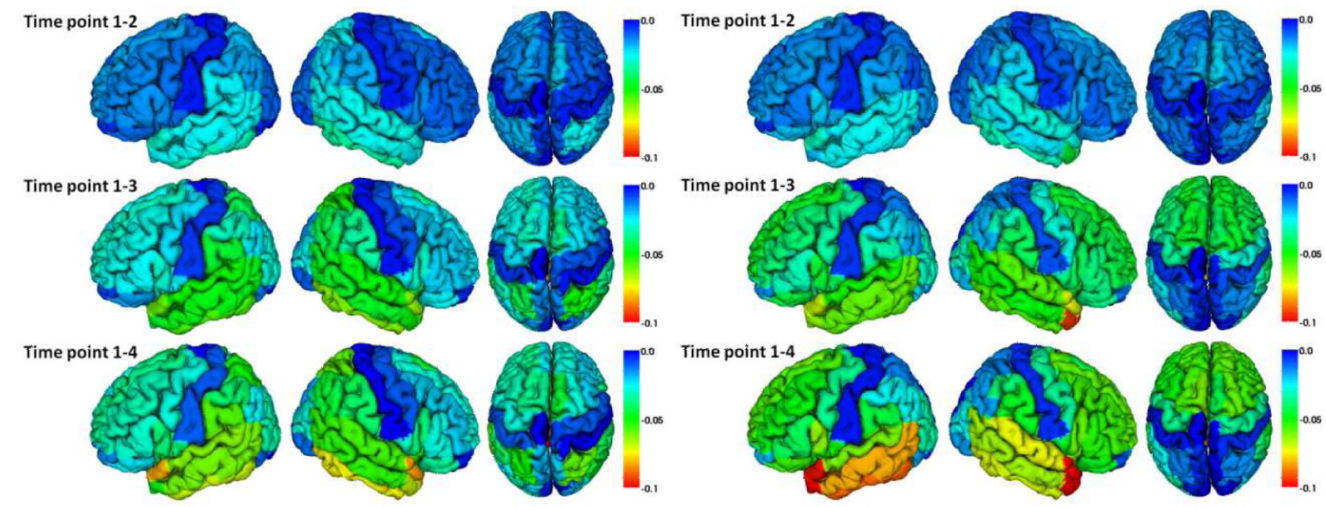

(c) P-MCI

(d) AD

Fig. 12.

The average changes of cortical thicknesses $(\mathrm{mm})$ of four groups of subjects betwwen time points 1-2, 1-3 and 1-4, measured in gyrus-based ROIs in the atlas space. The color bar is given on the right. 

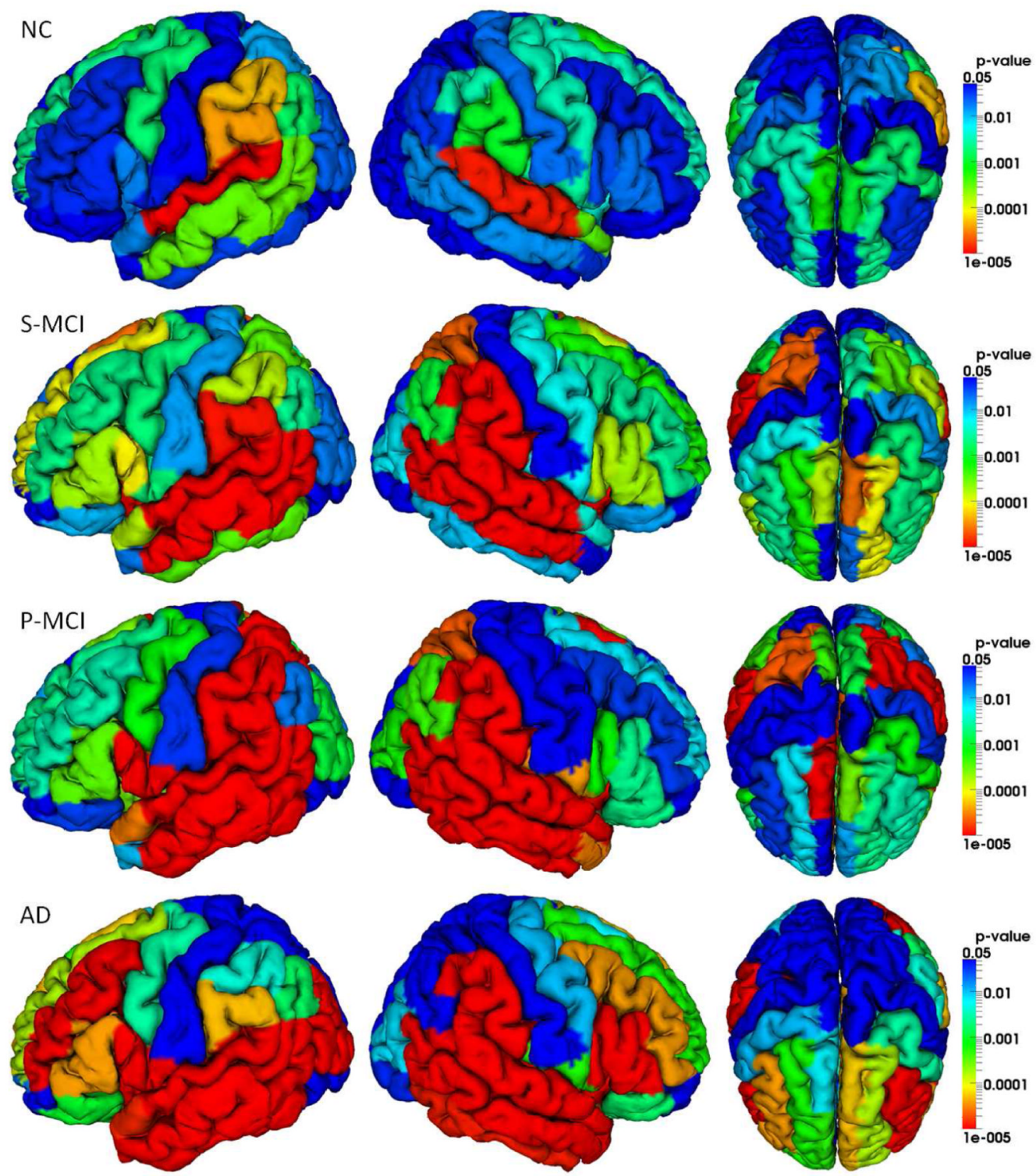

Fig. 13.

P-values of ROI-based cortical thickness thinning in 24 months in four groups of subjects. The color bar is provided on the right. 

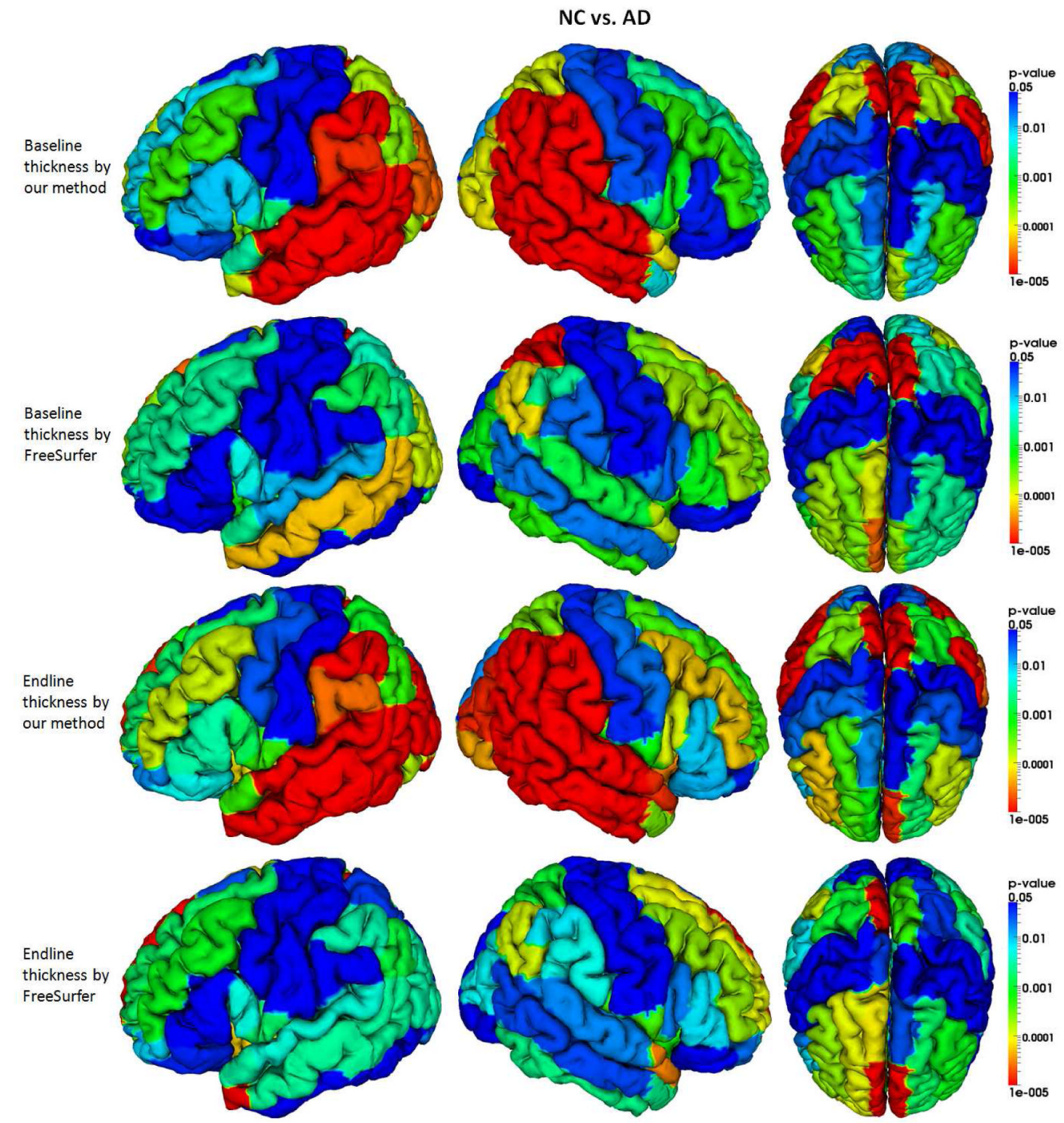

Fig. 14.

P-values of ROI-based cortical thickness difference between NC and AD groups by our method and FreeSurfer. The color bar is provided on the right. 

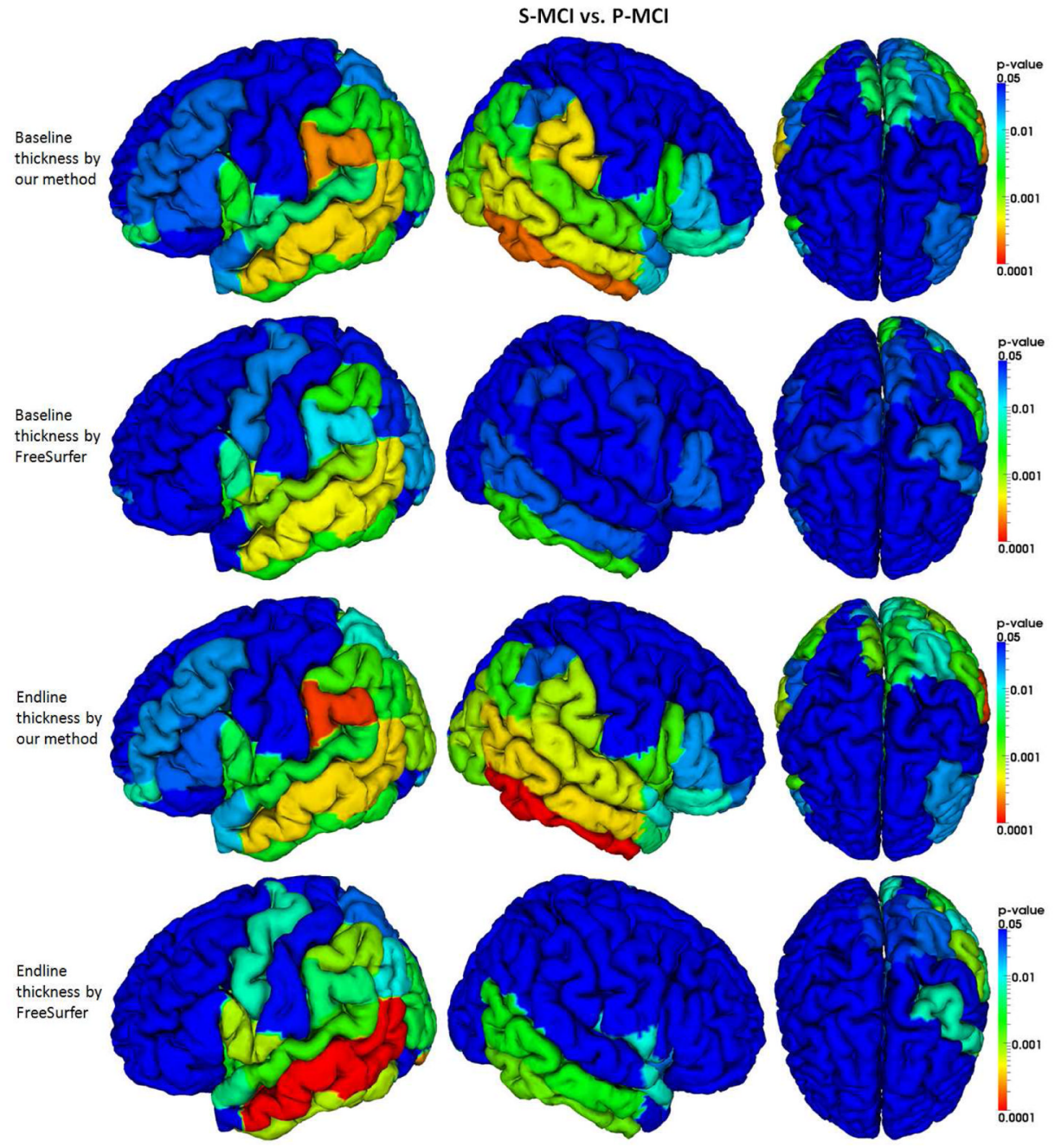

Fig. 15.

P-values of ROI-based cortical thickness difference between S-MCI and P-MCI groups by our method and FreeSurfer. The color bar is provided on the right. 


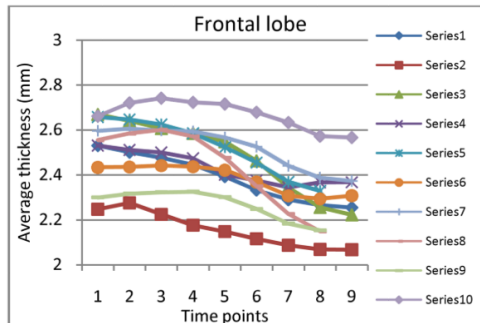

(a)

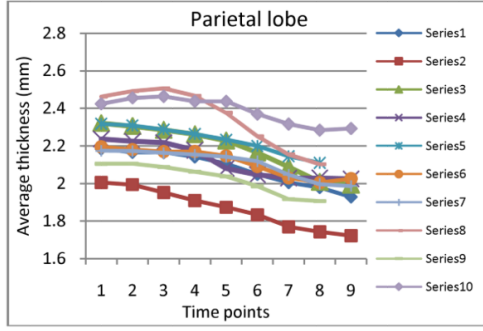

(c)



(e)

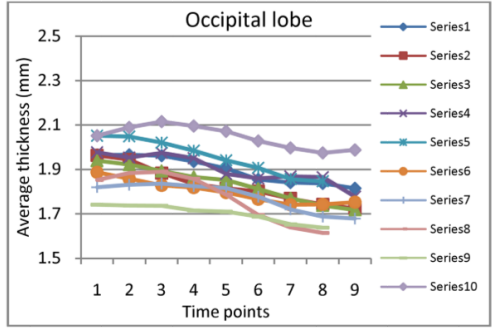

(g)

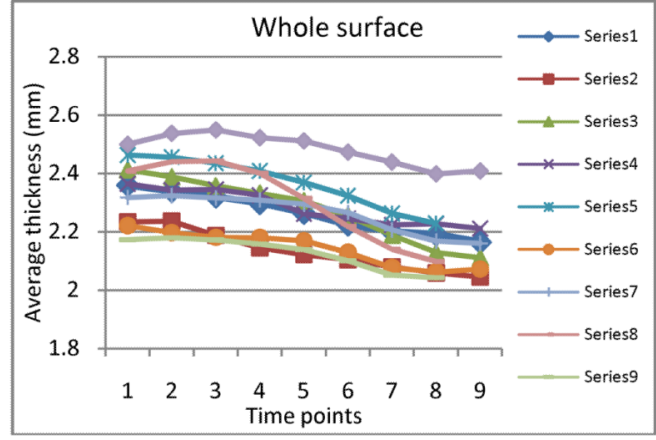

(i)

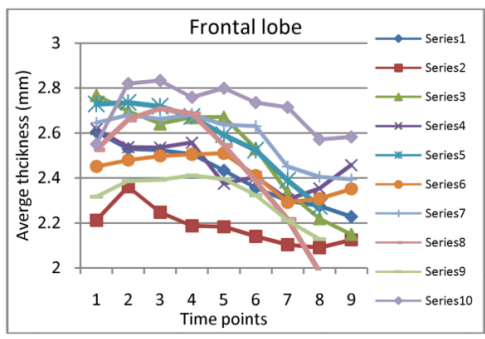

(b)

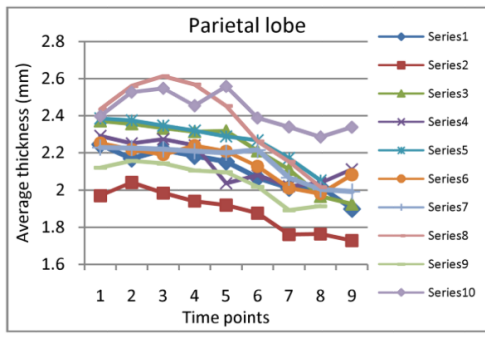

(d)

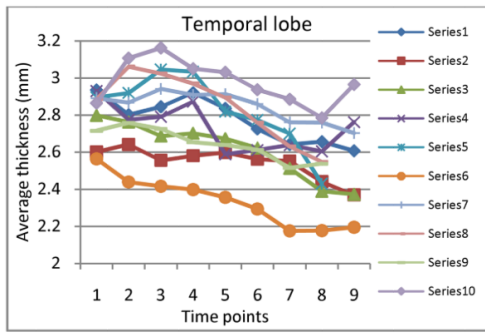

(f)

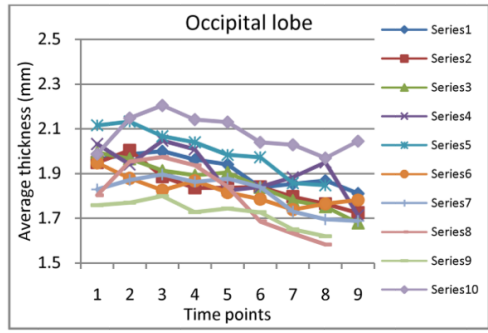

(h)

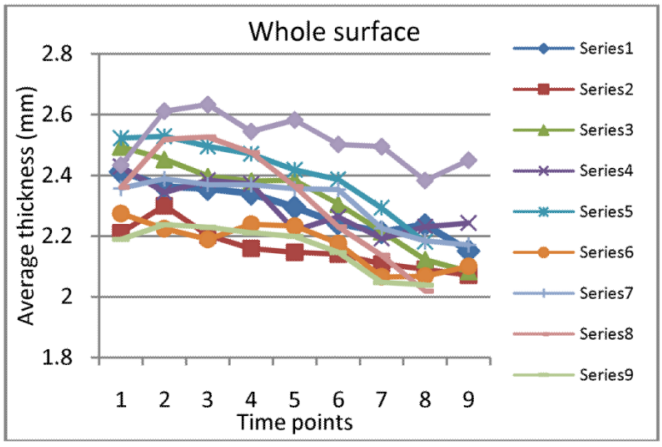

(j)

Fig. 16.

The longitudinal trajectories of average cortical thicknesses of four lobes on left hemipsheres and whole surfaces of 10 elderly subjects, each with 8 or 9 time points. One series indicates one subject. Figures (a), (c), (e), (g) and (i) show the results by our method 
with temporal constraint, while figures (b), (d), (f), (h) and (j) show the results by our method without temporal constraint. As we can see, the results by our method with temporal constraint are much more consistent and smoother than those by our method without temporal constraint. 
